INSTITUT NATIONAL DE RECHERCHE EN INFORMATIQUE ET EN AUTOMATIQUE

Behavior of upwind scheme in the low Mach number limit : III. Preconditioned dissipation for a five equation two phase model

\author{
Hervé Guillard — Angelo Murrone
}

$\mathbf{N}^{\circ} 5342$

October 21st 2004

Thème NUM

apport

de recherche 



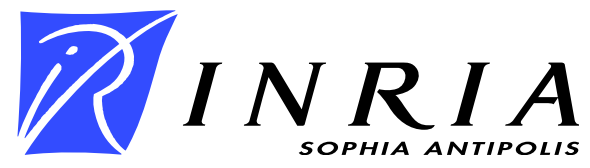

\title{
Behavior of upwind scheme in the low Mach number limit : III. Preconditioned dissipation for a five equation two phase model
}

\author{
Hervé Guillard* , Angelo Murrone ${ }^{\dagger}$ \\ Thème NUM — Systèmes numériques \\ Projet SMASH \\ Rapport de recherche $\mathrm{n}^{\circ} 5342$ - October 21st 2004 - 32 pages
}

\begin{abstract}
For single phase fluid models, like the Euler equations of compressible gas dynamics, upwind finite volume schemes suffer from a loss of accuracy when computing flows in the near incompressible regime. Preconditioning of the numerical dissipation is necessary to recover results consistent with the asymptotic behaviour of the continuous model. In this paper, we examine this situation for a two phase flow model. We show that as in the single phase case, the numerical approximation has to be done carefully in the near incompressible regime. We propose to adapt the preconditioning strategy used for single phase problems and present numerical results that show the efficiency of this approach
\end{abstract}

Key-words: Low Mach number limit, Asymptotic analysis, Preconditioning, Two phase flow model, Interface problems, Finite Volume schemes.

\footnotetext{
* Herve.Guillard@sophia.inria.fr - Projet SMASH

$\dagger$ Angelo.Murrone@sophia.inria.fr - Projet SMASH et CEA Cadarache, 13108 Saint-Paul-Lez-Durance (France)
} 


\section{Sur le comportement des schémas décentrés dans la limite des faibles nombres de Mach : III. Dissipation préconditionnée pour un modèle à cinq équations d'écoulements diphasiques}

Résumé : Pour les modèles d'écoulements monofluide, comme les équations d'Euler de la dynamique des gaz, les schémas décentrés de volumes finis souffrent d'une perte de précision lorsque on les applique au calcul d'écoulement fortement subsoniques. Le préconditionnement de la dissipation numérique est alors nécessaire pour retrouver des résultats consistants avec le comportement asymptotique des modèles continus. Dans ce travail, on examine la même situation pour un modèle d'écoulement diphasique. On montre que comme pour les écoulements mono-fluide, l'approximation numérique doit être adaptée pour pouvoir calculer des écoulements très subsonique. Nous proposons d'utiliser la même strategie de préconditionnement de la dissipation numérique que dans le cas mono-fluide et nous présentons des résultats numériques qui montrent l'efficacité de cette approche.

Mots-clés : Limite des faibles nombres de Mach, Analyse asymptotique, Préconditionnement, Modèles diphasiques, Problèmes d'interfaces, Schemas Volumes Finis. 


\section{Acknowledgements}

Angelo Murrone would like to thank his family for their constant support.

$\mathrm{RR} \mathrm{n}^{\circ} 5342$ 


\section{Contents}

1 Introduction $\quad 6$

$\begin{array}{lll}2 & \text { The continuous problem } & 7\end{array}$

3 Numerical approximation $\quad 11$

3.1 Preconditioned Riemann problem . . . . . . . . . . . . . . . . . . . . 12

3.2 Implicit linearized scheme . . . . . . . . . . . . . . . . . . . . . 15

3.3 Extension to second order space accuracy . . . . . . . . . . . . . . 17

4 Numerical results $\quad 19$

4.1 Bubble Ascension . . . . . . . . . . . . . . . . . . . . . . . . . . . . . . . 19

4.2 Broken Dam Problem . . . . . . . . . . . . . . . . . . . 22

5 Conclusion $\quad 22$

$\begin{array}{ll}\text { A Jacobian matrix } & 25\end{array}$

B Expression of the matrices $T$ and $T^{-1} \quad \mathbf{2 8}$ 


\section{List of Figures}

1 Definition of upwind and downwind triangle associated to edge $[\mathbf{i}, \mathbf{j}] \quad 18$

2 Bubble ascension : Isovalues of the volume fraction for the $100 \mathrm{x}$ 100 mesh computation : classical upwind scheme . . . . . . . 20

3 Bubble ascension : Isovalues of the volume fraction for the $100 \mathrm{x}$ 100 mesh computation : preconditioned scheme . . . . . . . . 20

4 Bubble ascension : Isovalues of the volume fraction for the $400 \mathrm{x}$ 400 fine mesh explicit computation . . . . . . . . . . . . . . 21

5 Broken dam problem : Isovalues of the volume fraction : classical upwind scheme . . . . . . . . . . . . . . . . . 23

6 Broken dam problem : Isovalues of the volume fraction : preconditioned scheme . . . . . . . . . . . . . . . . . . 23

7 Comparison between numerical solutions of the classical and preconditioned scheme and experimental results for the broken dam problem. Front position (top) and height of the column (bottom). . 24 
H. Guillard \&3 A. Murrone

\section{Introduction}

Although two phase and multifluid flows are encountered in a large number of situations from hypersonic to almost zero velocity flows, in many cases, the computation of these flows is a low Mach problem. This is true for instance in the nuclear or petroleum industry in nominal conditions when one of the two phases is a liquid with very small compressibility coefficient forcing the flow velocity to be small. The modelling of these flows is an extremely difficult task and nowadays, there is no universal model to take into account all the experimental conditions but instead exists a large collection of different type of models. In this paper, we are interested in modelisations of the type described for instance in [13], [10] in which the convective part of the model is described by an hyperbolic system. This includes a large variety of different modelisations ranging from simple homogeneous [3] or drift flux models to sophisticated two pressure, two velocity models [10], [1], [11]. With a few exceptions, from a theoritical poinf of view, the behaviour for vanishing Mach number of these two-phase flows models is unknown. A consequence of this absence of understanding of the behaviour of these models when the Mach number goes to zero is that the numerical approximation methods used to solve them is generally based on standard finite volume or finite difference methods. The efficiency of these methods is well assessed for the numerical approximation of hyperbolic systems in transonic and supersonic regimes. However, for very subsonic flows, when these discretization methods are applied to standard one phase models as the Euler or Navier-Stokes equations, it is now well-known that they suffers from efficiency and accuracy problems. Actually, it has even been proved in the case of the Euler equations that the numerical approximations produced by standard finite volume (FV) schemes of upwind type does not converge to the correct incompressible limit [6], [5]. There is no reason to believe that the situation is different for hyperbolic two-phase flow models and that in the low Mach number regime, standard discretization can be used safely to compute these flows. Actually, for one of the simplest two-phase flows model, namely the Homogeneous Equilibrium Model, it has been shown in [3] and [16] that FV upwind schemes exhibit the same type of accuracy problems than in the one phase context. In this paper, we investigate this situation for a more complex two-phase flow model, namely the five-equation model introduced in [7] and [9]. This model has been used for detonation studies in [7] and [17]. In [9], we have shown that it can also be used for some low Mach interface and two-phase flows problems. Our aim, here is to show that in the low Mach number regime, the numerical approximation of this model has to be done carefully if one want to avoid a loss of accuracy. Fortunately, we will also show that due to the mathematical structure of this model, the same recipe than in the case of the one-phase Euler equations can be applied and that preconditioning the numerical dissipation allows to recover a correct asymptotic behaviour when the Mach number goes to zero.

The summary of this work is as follows. In section 2, we perform an aymptotic analysis of the model in the low Mach number limit. The purpose of this section is to identify the correct limit equation that the solutions satisfy when the Mach number goes to zero. Using a single time asymptotic analysis, we show that for this incompressible limit, as for the one-phase Euler equations, pressure fluctuations scales with the square of the Mach number. 
Then in section 3, we propose an implicit numerical scheme based on a VFRoe-ncv solver [2] for its numerical approximation. This numerical scheme uses the solution of a preconditioned Riemann solver to enable a correct asymptotic behaviour of the numerical solution. Following the recipe proposed for the Euler equations in [6] and [5], this preconditioned Riemann solver is built using the close similarity between the mathematical structure of this model and the one of the one-phase Euler equations. Finally in section 4, we present a set of numerical experiments which show that this preconditioning strategy allows to recover accurate results when computing low Mach number flows with this model.

\section{The continuous problem}

The purpose of this section is to identify the limit equations and the asymptotic behavior of the solutions of a two phase one-velocity, one pressure model in the limit of vanishing low Mach number. Although this model describes the two phase medium by a single velocity and a single pressure, it retains two phase densities and a volume fraction for the description of the thermodynamical state of the fluid. Consequently, this model possesses two entropies (and two temperatures). This allows a richer description of the fluid than with the classical multicomponent Euler equations (that possesses a single temperature) and can have some advantages when the thermodynamics of the two fluids are very different. In term of conservative variables ${ }^{t}\left(\alpha_{1} \rho_{1}, \alpha_{2} \rho_{2}, \rho \boldsymbol{u}, \rho e, \alpha_{1}\right)$, this system can be written :

$$
\begin{cases}\frac{\partial \alpha_{1} \rho_{1}}{\partial t}+\operatorname{div}\left(\alpha_{1} \rho_{1} \boldsymbol{u}\right) & =0 \\ \frac{\partial \alpha_{2} \rho_{2}}{\partial t}+\operatorname{div}\left(\alpha_{2} \rho_{2} \boldsymbol{u}\right) & =0 \\ \frac{\partial \rho \boldsymbol{u}}{\partial t}+\operatorname{div}(\rho \boldsymbol{u} \otimes \boldsymbol{u})+\nabla p & =0 \\ \frac{\partial \rho e}{\partial t}+\operatorname{div}(\rho e+p) \boldsymbol{u} & =0 \\ \frac{\partial \alpha_{1}}{\partial t}+\boldsymbol{u} \cdot \nabla \alpha_{1} & =\alpha_{1} \alpha_{2} \frac{\rho_{2} a_{2}^{2}-\rho_{1} a_{1}^{2}}{\sum_{k=1}^{2} \alpha_{k^{\prime}} \rho_{k} a_{k}^{2}} \operatorname{div} \boldsymbol{u}\end{cases}
$$

Notations are classical. $\alpha_{k}$ are the volume fractions, $\rho_{k}$ the phase densities, $\boldsymbol{u}$ the vector velocity, and $p$ the pressure. Then $\rho=\sum_{k=1}^{2} \alpha_{k} \rho_{k}$ stands for the mixture density and $e$ the specific total energy is defined by $e=\varepsilon+\boldsymbol{u}^{2} / 2$ while the specific internal energy $\varepsilon$ is given by the relation $\rho \varepsilon=\sum_{k=1}^{2} \alpha_{k} \rho_{k} \varepsilon_{k}\left(p, \rho_{k}\right)$.

This model have been proposed for instance in [13], [17], [9] and can be obtained from an asymptotic analysis in the limit of zero relaxation time of the Baer-Nunziato two velocity,

RR $\mathrm{n}^{\circ} 5342$ 
two pressure model (see for instance [9]). Its mathematical properties are studied in [9] where it is shown that its structure is very close to the one of the one-phase Euler equations. Our aim, now is to perform an asymptotic analysis of this system when the Mach number tends to zero. Before that, let us recall the situation for the one-phase Euler equations : if the initial pressure field scales with the square of the Mach number : $p(\boldsymbol{x}, 0)=p^{0}+M_{*}^{2} p^{2}(\boldsymbol{x})$, and if the velocity at time $t=0$ is close to a divergence free field in the sense that $\boldsymbol{u}(\boldsymbol{x}, 0)=$ $\boldsymbol{u}^{0}(\boldsymbol{x})+M_{*} \boldsymbol{u}^{1}(\boldsymbol{x})$ with $\operatorname{div} \boldsymbol{u}^{0}=0$, then it is known that solutions of the Euler equations for compressible flows remain uniformly bounded as the Mach number tends to zero, and the limit solutions satisfy the equations for incompressible flows :

$$
\left\{\begin{array}{l}
\rho\left(\frac{\partial \boldsymbol{u}}{\partial t}+\operatorname{div}(\boldsymbol{u} \otimes \boldsymbol{u})\right)+\nabla \pi=0 \\
\operatorname{div}(\boldsymbol{u})=0
\end{array}\right.
$$

In the sequel, we will establish the same type of results for the model (1). For the sake of simplicity, we first rewrite system (1) using the pressure as independant variable instead of the total energy. The equation governing the evolution of the pressure is

$$
\frac{\partial p}{\partial t}+\boldsymbol{u} \cdot \nabla p+\rho \hat{a}^{2} \operatorname{div} \boldsymbol{u}=0
$$

In this last equation, we have introduced the averaged sound speed $\hat{a}$ defined by (see for instance [13], [17], [9])

$$
\frac{1}{\rho \hat{a}^{2}}=\sum_{k=1}^{2} \frac{\alpha_{k}}{\rho_{k} a_{k}^{2}}
$$

Formula (4) is the celebrated Wallis (or Woods) equilibrium sound speed well-known in the two-phase flow litterature (see e.g [13]).

The first step of the analysis is to perform a change a variables using non-dimensional variables instead of dimensional ones. Let $\rho_{\text {ref }}=\max [\rho(\boldsymbol{x}, 0)], u_{r e f}^{2}=\max \left[\boldsymbol{u}^{2}(\boldsymbol{x}, 0)\right]$ and let the sound speed scale $\hat{a}_{r e f}$ be defined by : $\hat{a}_{r e f}=\max [\hat{a}(\boldsymbol{x}, 0)]$.

Introducing the non-dimensionalized variables :

$$
\tilde{\rho}_{k}=\frac{\rho_{k}}{\rho_{\text {ref }}} \quad \tilde{\boldsymbol{u}}=\frac{\boldsymbol{u}}{u_{\text {ref }}} \quad \tilde{p}=\frac{p}{\rho_{\text {ref }} a_{\text {ref }}^{2}} \quad \tilde{\alpha}_{k}=\alpha_{k} \quad \tilde{\boldsymbol{x}}=\frac{\boldsymbol{x}}{\delta_{\text {ref }}} \quad \tilde{t}=\frac{t u_{\text {ref }}}{\delta_{\text {ref }}}
$$


with $\delta_{\text {ref }}$ an arbitrary length scale, (1) becomes:

$$
\begin{cases}\frac{\partial \tilde{\alpha}_{1} \tilde{\rho}_{1}}{\partial t}+\operatorname{div}\left(\tilde{\alpha}_{1} \tilde{\rho}_{1} \tilde{\boldsymbol{u}}\right) & =0 \\ \frac{\partial \tilde{\alpha}_{2} \tilde{\rho}_{2}}{\partial t}+\operatorname{div}\left(\tilde{\alpha}_{2} \tilde{\rho}_{2} \tilde{\boldsymbol{u}}\right) & =0 \\ \frac{\partial \tilde{\rho} \tilde{\boldsymbol{u}}}{\partial t}+\operatorname{div}(\tilde{\rho} \tilde{\boldsymbol{u}} \otimes \tilde{\boldsymbol{u}})+\frac{1}{M_{*}^{2}} \nabla \tilde{p} & =0 \\ \frac{\partial \tilde{p}}{\partial t}+\tilde{\boldsymbol{u}} \cdot \nabla \tilde{p}+\tilde{\rho} \tilde{a}^{2} \operatorname{div} \tilde{\boldsymbol{u}} & =0 \\ \frac{\partial \tilde{\alpha}_{1}}{\partial t}+\tilde{\boldsymbol{u}} \cdot \nabla \tilde{\alpha}_{1} & =\tilde{\alpha}_{1} \tilde{\alpha}_{2} \frac{\tilde{\rho}_{2} \tilde{a}_{2}^{2}-\tilde{\rho}_{1} \tilde{a}_{1}^{2}}{\sum_{k=1}^{2} \tilde{\alpha}_{k^{\prime}} \tilde{\rho}_{k} \tilde{a}_{k}^{2}} \operatorname{div} \tilde{\boldsymbol{u}}\end{cases}
$$

where $M_{*}=u_{r e f} / a_{r e f}$ is the reference Mach number.

And we now look for solution of system (6) in the form of asymptotic expansion in power of the Mach number $M_{*}$ :

$$
(\tilde{.})=(\tilde{.})^{0}+M_{*}(\tilde{\sim})^{1}+M_{*}^{2}(\tilde{\sim})^{2}+\mathcal{O}\left(M_{*}^{3}\right)
$$

Introducing these expressions into system (6) and collecting terms with equal power of $M_{*}$ we obtain at order $1 / M_{*}^{2}$ and $1 / M_{*}$ (in the sequel, we have dropped the subscripts $\sim$ for convenience)

$$
\left\{\begin{array}{l}
\nabla p^{0}=0 \\
\nabla p^{1}=0
\end{array}\right.
$$

These equations imply that the pressure is constant in space up to fluctuations of order $M_{*}^{2}$. Thus we may write :

$$
p(\boldsymbol{x}, t)=p^{0}(t)+M_{*}^{2} p^{2}(\boldsymbol{x}, t)
$$

$\mathrm{RR} \mathrm{n}^{\circ} 5342$ 
and this situation is then identical to the one obtained for the one-phase Euler equation. Then introducing these results in the order 1 system we get

$$
\begin{cases}\frac{\partial \alpha_{1}^{0} \rho_{1}^{0}}{\partial t}+\operatorname{div}\left(\alpha_{1}^{0} \rho_{1}^{0} \boldsymbol{u}^{0}\right) & =0 \\ \frac{\partial \alpha_{2}^{0} \rho_{2}^{0}}{\partial t}+\operatorname{div}\left(\alpha_{2}^{0} \rho_{2}^{0} \boldsymbol{u}^{0}\right) & =0 \\ \frac{\partial \rho^{0} \boldsymbol{u}^{0}}{\partial t}+\operatorname{div}\left(\rho^{0} \boldsymbol{u}^{0} \otimes \boldsymbol{u}^{0}\right)+\nabla p^{2} & =0 \\ \frac{d p^{0}}{d t}+\rho^{0}\left(\hat{a}^{0}\right)^{2} \operatorname{div} \boldsymbol{u}^{0} & =0 \\ \frac{\partial \alpha_{1}^{0}}{\partial t}+\boldsymbol{u}^{0} \cdot \nabla \alpha_{1}^{0} & =\alpha_{1}^{0} \alpha_{2}^{0} \frac{\rho_{2}^{0}\left(a_{2}^{0}\right)^{2}-\rho_{1}^{0}\left(a_{1}^{0}\right)^{2}}{\sum_{k=1}^{2} \alpha_{k^{\prime}}^{0} \rho_{k}^{0}\left(a_{k}^{0}\right)^{2}} \operatorname{div} \boldsymbol{u}^{0}\end{cases}
$$

To simplify, these equations, we note that in the presence of open boundaries, the thermodynamic pressure $p^{0}$ will be imposed and be equal to the exterior pressure. For the sake of simplicity, we assume that the exterior pressure does not change with time, and thus, the pressure $p^{0}$ will be a constant in space and time :

$$
\frac{d p^{E x t}}{d t}=\frac{d p^{0}}{d t}=0
$$

and the pressure equation (10.4) degenerates into :

$$
\operatorname{div} \boldsymbol{u}^{0}=0
$$

Again, this situation is totally identical to the one of the one-phase Euler equation. Now, introducing relation (12) into the mass conservation equations (10.1)-(10.2) and the volume fraction equation (10.5), we get :

$$
\frac{\partial \rho_{k}^{0}}{\partial t}+\boldsymbol{u}^{0} \cdot \nabla \rho_{k}^{0}=0 \quad \text { and } \quad \frac{\partial \alpha_{1}^{0}}{\partial t}+\boldsymbol{u}^{0} \cdot \nabla \alpha_{1}^{0}=0
$$

Assuming that all particle paths come from regions with the same phase densities, we conclude that $\rho_{k}^{0}=$ Cte and thus the set of equations that governs the evolution of the variables 
${ }^{t}\left(\alpha_{1} \rho_{1}, \alpha_{2} \rho_{2}, \rho \boldsymbol{u}, \rho e, \alpha_{1}\right)$ is asymptotically in the limit $M a \rightarrow 0$

$$
\left\{\begin{array}{l}
\rho_{1}=\text { Cte } \\
\rho_{2}=\text { Cte } \\
\frac{\partial \boldsymbol{u}}{\partial t}+\operatorname{div}(\boldsymbol{u} \otimes \boldsymbol{u})+\frac{1}{\rho\left(\alpha_{1}\right)} \nabla p=0 \\
\operatorname{div} \boldsymbol{u}=0 \\
\frac{\partial \alpha_{1}}{\partial t}+\boldsymbol{u} \cdot \nabla \alpha_{1}=0
\end{array}\right.
$$

where the mixture density $\rho\left(\alpha_{1}\right)$ depends only to the volume fraction $\alpha_{1}$ which is simply advected at the velocity $\boldsymbol{u}$ of the flow. Note the close similarity with the one phase incompressible Euler equations (2).

\section{Numerical approximation}

System (1) is not a conservative system due to the evolution equation for the mass fraction

$$
\frac{\partial \alpha_{1}}{\partial t}+\boldsymbol{u} \cdot \nabla \alpha_{1}=\alpha_{1} \alpha_{2} \frac{\rho_{2} a_{2}^{2}-\rho_{1} a_{1}^{2}}{\sum_{k=1}^{2} \alpha_{k^{\prime}} \rho_{k} a_{k}^{2}} \operatorname{div} \boldsymbol{u}
$$

where $k^{\prime}=(2,1)$ for $k=(1,2)$. In the numerical approximation used in this paper, we re-write this equation as

$$
\frac{\partial \alpha_{1}}{\partial t}+\operatorname{div}\left(\alpha_{1} \boldsymbol{u}\right)+B(\boldsymbol{Q}) \operatorname{div} \boldsymbol{u}=0 \quad \text { with } \quad B(\boldsymbol{Q})=\frac{-\alpha_{1} \rho_{2} a_{2}^{2}}{\sum_{k=1}^{2} \alpha_{k^{\prime}} \rho_{k} a_{k}^{2}}
$$

Then let $\boldsymbol{Q}={ }^{t}\left(\alpha_{1} \rho_{1}, \alpha_{2} \rho_{2}, \rho \boldsymbol{u}, \rho e, \alpha_{1}\right)$ be the set of "conservative" ${ }^{1}$ variables. With this definition, the system (1) can be written as :

$$
\frac{\partial \boldsymbol{Q}}{\partial t}+\operatorname{div} \boldsymbol{F}(\boldsymbol{Q})+\operatorname{div} \boldsymbol{u} \boldsymbol{B}(\boldsymbol{Q})=0
$$

where $\boldsymbol{B}(\boldsymbol{Q})={ }^{t}(0,0,0,0, B(\boldsymbol{Q}))$. Integrating this equation on a cell $C_{i}$ gives :

$$
A_{i} \frac{\partial \boldsymbol{Q}_{i}}{\partial t}+\int_{\partial C_{i}} \boldsymbol{F}(\boldsymbol{Q}) \cdot \boldsymbol{n} d l+\int_{C_{i}} \boldsymbol{B}(\boldsymbol{Q}) \operatorname{div} \boldsymbol{u} d \Omega=0 \quad \text { for } \quad i \in\{1, \ldots, N\}
$$

\footnotetext{
${ }^{1}$ Although $\alpha$ is not a conserved quantity, we will use this terminology for convenience
} 
where $N$ is the number of cells and $A_{i}$ is the volume of the cell $C_{i}$. Defining $v(i)$ as the set of cells $C_{j}$ that share an edge with $C_{i}$ and defining $\partial C_{i j}=\partial C_{i} \cap \partial C_{j}$, we approximate (18) by the following expression :

$$
A_{i} \frac{\partial \boldsymbol{Q}_{i}}{\partial t}+\sum_{j \in v(i)} \int_{\partial C_{i j}} \boldsymbol{F}(\boldsymbol{Q}) \cdot \boldsymbol{n} d l+<\boldsymbol{B}>_{i} \sum_{j \in v(i)} \int_{\partial C_{i j}} \boldsymbol{u} \cdot \boldsymbol{n} d l=0
$$

where $\langle\boldsymbol{B}\rangle_{i}$ is some average of $\boldsymbol{B}$ on the cell $C_{i}$. In this work, we have used $\langle\boldsymbol{B}\rangle_{i}=$ $B\left(\boldsymbol{Q}_{i}\right)$ or $\left\langle\boldsymbol{B}>_{i}=\sum_{j \in v(i)} \boldsymbol{B}\left(\boldsymbol{Q}_{i j}^{*}\right) / \sum_{j \in v(i)} 1\right.$ with no noticeable difference. Then the surface integrals appearing in (19) are computed by a one-point formula to yield :

$$
A_{i} \frac{\partial \boldsymbol{Q}_{i}}{\partial t}+\sum_{j \in v(i)}\left\|\boldsymbol{n}_{i j}\right\|\left(<\boldsymbol{F}(\boldsymbol{Q}) . \boldsymbol{n}>_{i j}+<\boldsymbol{B}>_{i}<\boldsymbol{u} . \boldsymbol{n}>_{i j}\right)=0
$$

where $\boldsymbol{n}_{i j}=\int_{\partial C_{i j}} \boldsymbol{n} d l$ is the integral of the normal vector of the interface and $\langle\boldsymbol{F}(\boldsymbol{Q}) \cdot \boldsymbol{n}\rangle_{i j}$ $\left(\right.$ resp. $\left\langle\boldsymbol{u} . \boldsymbol{n}>_{i j}\right.$ ) denotes some averages of $\boldsymbol{F}(\boldsymbol{Q}) \cdot \boldsymbol{n}$ (resp. u.n ) on $\partial C_{i j}$. In this work, we use a VFRoe type solver [2] and define these average values by

$$
\begin{aligned}
& <\boldsymbol{F}(\boldsymbol{Q}) \cdot \boldsymbol{n}>_{i j}=\boldsymbol{F}\left(\boldsymbol{Q}_{i j}^{*}\right) \cdot \boldsymbol{\eta}_{i j} \\
& <\boldsymbol{u} . \boldsymbol{n}>_{i j}=\boldsymbol{u}_{i j}^{*} \cdot \boldsymbol{\eta}_{i j}
\end{aligned}
$$

where $\boldsymbol{\eta}_{i j}=\boldsymbol{n}_{i j} /\left\|\boldsymbol{n}_{i j}\right\|$ and $\boldsymbol{Q}_{i j}^{*}$ is the solution of an approximate Riemann solver between the states $\boldsymbol{Q}_{i}$ and $\boldsymbol{Q}_{j}$ and $\boldsymbol{u}_{i j}^{*} \cdot \boldsymbol{\eta}_{i j}$ is the corresponding normal velocity. In low Mach number situations, the correct definition of this Riemann problem is crucial for the accuracy of the numerical approximation. In the next section, we describe how this Riemann problem is set.

\subsection{Preconditioned Riemann problem}

In standard upwind method, the Riemann problem defining the state $\boldsymbol{Q}_{i j}^{*}$ is based on the original differential system (1). Thus, let $\boldsymbol{q}=\boldsymbol{q}(\boldsymbol{Q})$ denote some change of variables such that $R=\partial \boldsymbol{q} / \partial \boldsymbol{Q}$ is invertible and define a local basis $\left(\boldsymbol{\eta}_{L R}, \boldsymbol{\eta}_{L R}^{\perp}\right)$ of unit vectors respectively normal and tangential to the interface. In term of these new variables, the Riemann problem between the states $\boldsymbol{Q}_{i}=\boldsymbol{Q}_{L}$ and $\boldsymbol{Q}_{j}=\boldsymbol{Q}_{R}$ that will allow to compute $\boldsymbol{Q}_{i j}^{*}=\boldsymbol{Q}\left(x / t=0 ; \boldsymbol{Q}_{L}, \boldsymbol{Q}_{R}\right)$ is defined by :

$$
\left\{\begin{array}{l}
\frac{\partial \boldsymbol{q}}{\partial t}+A_{e}(\boldsymbol{q}) \frac{\partial \boldsymbol{q}}{\partial x}=0 \\
\boldsymbol{q}(x, 0)=\mid \begin{array}{lll}
\boldsymbol{q}_{L} & \text { if } & x<0 \\
\boldsymbol{q}_{R} & \text { if } & x>0
\end{array}
\end{array}\right.
$$

where $A_{e}=R .\left[\partial\left(\boldsymbol{F}(\boldsymbol{Q}) \cdot \boldsymbol{\eta}_{L R}\right) / \partial \boldsymbol{Q}\right] R^{-1}$. However, in the low Mach number limit, for the one-phase Euler equations, this strategy leads to numerical schemes that do not have the 
correct asymptotic behaviour. This situation is explained in detail in [5] where it is shown that the trouble comes from the fact that the interface pressure computed by the Riemann solver based on (22) contains pressure fluctuations of order Mach even if the initial data contain fluctuations that scale with the square of the Mach number. In [5], to overcome this diffculty, we proposed to solve instead of the Riemann problem (22) based on the original differential system, to solve a preconditioned Riemann problem. We propose here to apply the same strategy to the system (1). The transposition of this strategy to system (1) is greatly simplified by the fact that the mathematical structure of this model is very close to the one of the Euler equation. Actually, it is shown in [9] that in term of "entropic" variables $\tilde{\boldsymbol{q}}={ }^{t}\left(p, \boldsymbol{u}, s_{1}, s_{2}, Y_{1}\right)$ system (1) can be written as :

$$
\left\{\begin{array}{llll}
\frac{D p}{D t}+\rho \hat{a}^{2} \operatorname{div} \boldsymbol{u} & = & 0 & (23.1) \\
\frac{D \boldsymbol{u}}{D t}+\frac{1}{\rho} \nabla p & = & 0 \\
\frac{D s_{1}}{D t} & = & 0 & (23.2) \\
\frac{D s_{2}}{D t} & = & 0 \\
\frac{D Y_{1}}{D t} & = & 0
\end{array}\right.
$$

where we have introduced the notation $D / D t=\partial / \partial t+\boldsymbol{u} . \nabla$.

Using this form of the equations, it becomes obvious that the only change with the onephase Euler equation is that we have now three linearly degenerate fields instead of a single one. However, the formal structure of the two systems are identical. Therefore, extending the method used in [5], we define a "Turkel" preconditioner [14] by :

$$
P_{e}(\beta)=\operatorname{diag}\left(\beta^{2}, I d_{n}, 1,1,1\right)
$$

where $I d_{n}$ is the $n$-dimensional identity matrix ( $n$ is the space dimension) and $\beta$ a parameter of the order of the Mach number. For the one-phase Euler equations, we recall that the Turkel preconditioner in entropic variables is defined by $P_{e}(\beta)=\operatorname{diag}\left(\beta^{2}, I d_{n}, 1,\right)$. With this definition, as in [5],instead of solving (22), we will solve a preconditioned Riemann problem defined as :

$$
\left\{\begin{array}{l}
\frac{\partial \tilde{\boldsymbol{q}}}{\partial t}+P_{e}(\beta) A_{e}(<\tilde{\boldsymbol{q}}>) \frac{\partial \tilde{\boldsymbol{q}}}{\partial x}=0 \\
\tilde{\boldsymbol{q}}(x, 0)=\mid \begin{array}{lll}
\tilde{\boldsymbol{q}}_{L} & \text { if } & x<0 \\
\tilde{\boldsymbol{q}}_{R} & \text { if } & x>0
\end{array}
\end{array}\right.
$$


where $<.>=\left((.)_{L}+(.)_{R}\right) / 2$ denotes the arithmetic average between the states $(.)_{L}$ and $(.)_{R}$. Note that using $\beta=1$ i.e $P(\beta)=I d$ will simply result in a non-preconditioned scheme, this allows to recover a standard approximation for transonic flows.

The matrix $P_{e}(\beta) A_{e}(\tilde{\boldsymbol{q}})$ is given by

$$
P_{e}(\beta) A_{e}(\tilde{\boldsymbol{q}})=\left(\begin{array}{cccccc}
\beta^{2} v_{n} & \beta^{2} \rho \hat{a}^{2} & 0 & 0 & 0 & 0 \\
1 / \rho & v_{n} & 0 & 0 & 0 & 0 \\
0 & 0 & v_{n} I d_{n-1} & 0 & 0 & 0 \\
0 & 0 & 0 & v_{n} & 0 & 0 \\
0 & 0 & 0 & 0 & v_{n} & 0 \\
0 & 0 & 0 & 0 & 0 & v_{n}
\end{array}\right)
$$

where $v_{n}=\boldsymbol{u} . \boldsymbol{\eta}_{L R}$. This matrix is diagonalizable. In 2-D, for instance, forming the characteristic equation $\left(v_{n}-\lambda\right)^{4}\left(\lambda^{2}-\left(1+\beta^{2}\right) v_{n} \lambda+\beta^{2}\left(v_{n}^{2}-\hat{a}^{2}\right)\right)=0$, we get three distinct real eigenvalues :

$$
\left\{\begin{array}{l}
\lambda_{1}(\tilde{\boldsymbol{q}})=\frac{1}{2}\left[\left(1+\beta^{2}\right) v_{n}-\sqrt{X}\right] \\
\lambda_{2}(\tilde{\boldsymbol{q}})=\lambda_{3}(\tilde{\boldsymbol{q}})=\lambda_{4}(\tilde{\boldsymbol{q}})=\lambda_{5}(\tilde{\boldsymbol{q}})=v_{n} \\
\lambda_{6}(\tilde{\boldsymbol{q}})=\frac{1}{2}\left[\left(1+\beta^{2}\right) v_{n}+\sqrt{X}\right]
\end{array}\right.
$$

where we have introduced the parameter $X=\left[\left(1-\beta^{2}\right) v_{n}\right]^{2}+4 \beta^{2} \hat{a}^{2}$. The associated right eigenvectors $r_{i}(\tilde{\boldsymbol{q}})$ (for $\left.i \in\{1, \ldots, 6\}\right)$, that verify the relation $P_{e}(\beta) A_{e}(\tilde{\boldsymbol{q}}) r_{i}(\tilde{\boldsymbol{q}})=\lambda_{i}(\tilde{\boldsymbol{q}}) r_{i}(\tilde{\boldsymbol{q}})$ can be respectively choosen as :

$$
\begin{array}{ll}
r_{1}(\tilde{\boldsymbol{q}})=\left(\begin{array}{c}
1 \\
\frac{-s}{\beta^{2} \rho \hat{a}^{2}} \\
0 \\
0 \\
0 \\
0
\end{array}\right) & r_{2}(\tilde{\boldsymbol{q}})=\left(\begin{array}{c}
0 \\
0 \\
1 \\
0 \\
0 \\
0
\end{array}\right) \quad r_{3}(\tilde{\boldsymbol{q}})=\left(\begin{array}{c}
0 \\
0 \\
0 \\
1 \\
0 \\
0
\end{array}\right) \\
r_{4}(\tilde{\boldsymbol{q}})=\left(\begin{array}{c}
0 \\
0 \\
0 \\
0 \\
1 \\
0
\end{array}\right) & r_{5}(\tilde{\boldsymbol{q}})=\left(\begin{array}{c}
0 \\
0 \\
0 \\
0 \\
0 \\
1
\end{array}\right) \quad r_{6}(\tilde{\boldsymbol{q}})=\left(\begin{array}{c}
1 \\
\frac{-r}{\beta^{2} \rho \hat{a}^{2}} \\
0 \\
0 \\
0 \\
0
\end{array}\right)
\end{array}
$$

where $r=\lambda_{1}-v_{n}$ and $s=\lambda_{6}-v_{n}$. We denote also by $l_{i}(\boldsymbol{q})$ (for $i \in\{1, \ldots, 6\}$ ) the left eigenvectors which obey the relation ${ }^{t} P_{e}(\beta) A_{e}(\tilde{\boldsymbol{q}}) l_{i}(\boldsymbol{q})=\lambda_{i}(\boldsymbol{q}) l_{i}(\boldsymbol{q})$. After normalization of 
left and right eigenvectors to have ${ }^{t} l_{i}(\boldsymbol{q}) \cdot r_{j}(\boldsymbol{q})=\delta_{i j}$, we get :

$$
\begin{array}{cc}
l_{1}(\tilde{\boldsymbol{q}})=\frac{-1}{\sqrt{X}}\left(\begin{array}{c}
r \\
\beta^{2} \rho \hat{a}^{2} \\
0 \\
0 \\
0 \\
0
\end{array}\right) & l_{2}(\tilde{\boldsymbol{q}})=\left(\begin{array}{l}
0 \\
0 \\
1 \\
0 \\
0 \\
0
\end{array}\right) \quad l_{3}(\tilde{\boldsymbol{q}})=\left(\begin{array}{l}
0 \\
0 \\
0 \\
1 \\
0 \\
0
\end{array}\right) \\
l_{4}(\tilde{\boldsymbol{q}})=\left(\begin{array}{c}
0 \\
0 \\
0 \\
0 \\
1 \\
0
\end{array}\right) & l_{5}(\tilde{\boldsymbol{q}})=\left(\begin{array}{l}
0 \\
0 \\
0 \\
0 \\
0 \\
1
\end{array}\right) \quad l_{6}(\tilde{\boldsymbol{q}})=\frac{1}{\sqrt{X}}\left(\begin{array}{c}
s \\
\beta^{2} \rho \hat{a}^{2} \\
0 \\
0 \\
0 \\
0
\end{array}\right)
\end{array}
$$

Thus, the approximate state in term of "entropic" variables at the interface, i.e on $x / t=0$, is given by :

$$
\tilde{\boldsymbol{q}}_{L R}^{*}=\frac{1}{2}\left[\tilde{\boldsymbol{q}}_{L}+\tilde{\boldsymbol{q}}_{R}+\left[r_{i}\right] \operatorname{sgn}\left[\operatorname{diag}\left(\lambda_{i}\right)(<\tilde{\boldsymbol{q}}>)\right]^{t}\left[l_{i}\right] \Delta \tilde{\boldsymbol{q}}_{L R}\right]
$$

where $\Delta \tilde{\boldsymbol{q}}_{L R}=\tilde{\boldsymbol{q}}_{L}-\tilde{\boldsymbol{q}}_{R}$ and $\left[r_{i}\right],\left[l_{i}\right]$ denote the matrices whose column vectors are respectively the right and left eigenvectors (28) and (29).

\subsection{Implicit linearized scheme}

Let us define

$$
\boldsymbol{\psi}\left(\boldsymbol{Q}_{i}, \boldsymbol{Q}_{j}\right)=\boldsymbol{F}\left(\boldsymbol{Q}_{i j}^{*}\right) \cdot \boldsymbol{\eta}_{i j}+\boldsymbol{B}\left(\boldsymbol{Q}_{i}\right) \boldsymbol{u}_{i j}^{*} \cdot \boldsymbol{\eta}_{i j}
$$

where $\boldsymbol{Q}_{i j}^{*}$ is the conservative variable corresponding to the state $\tilde{\boldsymbol{q}}_{i j}^{*}$ defined by equation (30) in term of "entropic" variables. A fully implicit first-order scheme using this expression would be defined by :

$$
A_{i} \frac{\boldsymbol{Q}_{i}^{n+1}-\boldsymbol{Q}_{i}^{n}}{\Delta t}+\sum_{j \in v(i)}\left\|\boldsymbol{n}_{i j}\right\| \boldsymbol{\psi}\left(\boldsymbol{Q}_{i}^{n+1}, \boldsymbol{Q}_{j}^{n+1}\right)=0
$$

However, this expression defines a non-algebraic system for $\boldsymbol{Q}_{i}$. A linear scheme of the same order of accuracy can be obtained by linearizing this expression around the state $\boldsymbol{Q}^{n}$ thanks to a Taylor developement of the first order. This gives the linear first-order scheme

$$
\begin{aligned}
& A_{i} \frac{\boldsymbol{Q}_{i}^{n+1}-\boldsymbol{Q}_{i}^{n}}{\Delta t}+\sum_{j \in v(i)}\left\|\boldsymbol{n}_{i j}\right\|\left[\boldsymbol{\psi}\left(\boldsymbol{Q}_{i}^{n}, \boldsymbol{Q}_{j}^{n}\right)\right. \\
& \left.+\frac{\partial \boldsymbol{\psi}\left(\boldsymbol{Q}_{i}^{n}, \boldsymbol{Q}_{j}^{n}\right)}{\partial \boldsymbol{Q}_{i}^{n}}\left(\boldsymbol{Q}_{i}^{n+1}-\boldsymbol{Q}_{i}^{n}\right)+\frac{\partial \boldsymbol{\psi}\left(\boldsymbol{Q}_{i}^{n}, \boldsymbol{Q}_{j}^{n}\right)}{\partial \boldsymbol{Q}_{j}^{n}}\left(\boldsymbol{Q}_{j}^{n+1}-\boldsymbol{Q}_{j}^{n}\right)\right]=0
\end{aligned}
$$

$\mathrm{RR} \mathrm{n}^{\circ} 5342$ 
Neglecting the derivative of the non-conservative term $\boldsymbol{B}\left(\boldsymbol{Q}_{i}\right)$, we obtain

$$
\left\{\begin{aligned}
\frac{\partial \boldsymbol{\psi}\left(\boldsymbol{Q}_{i}^{n}, \boldsymbol{Q}_{j}^{n}\right)}{\partial \boldsymbol{Q}_{i}^{n}} \simeq\left[\frac{\partial \boldsymbol{F}\left(\boldsymbol{Q}_{i j}^{*}\right) \cdot \boldsymbol{\eta}_{i j}}{\partial \boldsymbol{Q}_{i j}^{*}}+\boldsymbol{B}\left(\boldsymbol{Q}_{i}^{n}\right) \frac{\partial \boldsymbol{u}_{i j}^{*} \cdot \boldsymbol{\eta}_{i j}}{\partial \boldsymbol{Q}_{i j}^{*}}\right] \frac{\partial \boldsymbol{Q}_{i j}^{*}}{\partial \boldsymbol{Q}_{i}^{n}} \\
\frac{\partial \boldsymbol{\psi}\left(\boldsymbol{Q}_{i}^{n}, \boldsymbol{Q}_{j}^{n}\right)}{\partial \boldsymbol{Q}_{j}^{n}} \simeq\left[\frac{\partial \boldsymbol{F}\left(\boldsymbol{Q}_{i j}^{*}\right) \cdot \boldsymbol{\eta}_{i j}}{\partial \boldsymbol{Q}_{i j}^{*}}+\boldsymbol{B}\left(\boldsymbol{Q}_{i}^{n}\right) \frac{\partial \boldsymbol{u}_{i j}^{*} \cdot \boldsymbol{\eta}_{i j}}{\partial \boldsymbol{Q}_{i j}^{*}}\right] \frac{\partial \boldsymbol{Q}_{i j}^{*}}{\partial \boldsymbol{Q}_{j}^{n}}
\end{aligned}\right.
$$

The expression for the Jacobian matrix $\frac{\partial \boldsymbol{F}\left(\boldsymbol{Q}_{i j}^{*}\right) \cdot \boldsymbol{\eta}_{i j}}{\partial \boldsymbol{Q}_{i j}^{*}}$ is given in Appendix A and the only remaining difficulty is to define $\frac{\partial \boldsymbol{Q}_{i j}^{*}}{\partial \boldsymbol{Q}_{i}}$ and $\frac{\partial \boldsymbol{Q}_{i j}^{*}}{\partial \boldsymbol{Q}_{j}}$. In this work, to approximate these derivatives, we have considered that the conservative variable $\boldsymbol{Q}_{L R}^{*}=\boldsymbol{Q}\left(\tilde{\boldsymbol{q}}_{L R}^{*}\right)$ corresponding to the "entropic" variable $\tilde{\boldsymbol{q}}_{L R}^{*}$ defined by expression (30) can be approximated by :

$$
\boldsymbol{Q}_{L R}^{*} \simeq \frac{1}{2}\left[\boldsymbol{Q}_{L}+\boldsymbol{Q}_{R}+\theta^{-1} R^{-1}\left[r_{i}\right] \operatorname{sgn}\left[\operatorname{diag}\left(\lambda_{i}\right)(<\tilde{\boldsymbol{q}}>)\right]^{t}\left[l_{i}\right] R \theta \Delta \boldsymbol{Q}_{L R}\right]
$$

where $R=\partial \tilde{\boldsymbol{q}} / \partial \boldsymbol{Q}$ and $R^{-1}=\partial \boldsymbol{Q} / \tilde{\partial \boldsymbol{q}}$ are the change of variables matrices between conservative variables $\boldsymbol{Q}$ and "entropic" ones $\boldsymbol{q}$ while $\theta$ and $\theta^{-1}$ are the rotation matrices that transform $\boldsymbol{Q}$ in the global basis to $\theta \boldsymbol{Q}={ }^{t}\left(\alpha_{1} \rho_{1}, \alpha_{2} \rho_{2}, \rho v_{n}, \rho v_{t}, \rho e, \alpha_{1}\right)$ in the local basis $\left(\boldsymbol{\eta}, \boldsymbol{\eta}^{\perp}\right)$ with $v_{n}=\boldsymbol{u} . \boldsymbol{\eta}, v_{t}=\boldsymbol{u} . \boldsymbol{\eta}^{\perp}$ the normal and tangential components of the vector velocity to the local cell interface. With this approximation, we get

$$
\left\{\begin{array}{l}
\frac{\partial \boldsymbol{Q}_{i j}^{*}}{\partial \boldsymbol{Q}_{i}^{n}} \simeq \frac{1}{2}\left[I d+T^{-1} \operatorname{sgn}\left[\operatorname{diag}\left(\lambda_{i}\right)(<\tilde{\boldsymbol{q}}>)\right] T\right] \\
\frac{\partial \boldsymbol{Q}_{i j}^{*}}{\partial \boldsymbol{Q}_{j}^{n}} \simeq \frac{1}{2}\left[I d-T^{-1} \operatorname{sgn}\left[\operatorname{diag}\left(\lambda_{i}\right)(<\tilde{\boldsymbol{q}}>)\right] T\right]
\end{array}\right.
$$

where $I d$ stands for the identity matrix and $T$ and $T^{-1}$ are the matrices $T={ }^{t}\left[l_{i}\right] R \theta$ and $T^{-1}=\theta^{-1} R^{-1}\left[r_{i}\right]$. The expression of these matrices is given in Appendix B. Finally, the first order implicit backward scheme can be written as :

$$
\begin{aligned}
& A_{i} \frac{\boldsymbol{Q}_{i}^{n+1}-\boldsymbol{Q}_{i}^{n}}{\Delta t}+\sum_{j \in v(i)}\left\|\boldsymbol{n}_{i j}\right\|\left[\boldsymbol{F}\left(\boldsymbol{Q}_{i j}^{*}\right) \cdot \boldsymbol{\eta}_{i j}+\boldsymbol{B}\left(\boldsymbol{Q}_{i}\right) \boldsymbol{u}_{i j}^{*} \cdot \boldsymbol{\eta}_{i j}\right. \\
& +\frac{1}{2}\left[\frac{\partial \boldsymbol{F}\left(\boldsymbol{Q}_{i j}^{*}\right) \cdot \boldsymbol{\eta}_{i j}}{\partial \boldsymbol{Q}_{i j}^{*}}+\boldsymbol{B}\left(\boldsymbol{Q}_{i}^{n}\right) \frac{\partial \boldsymbol{u}_{i j}^{*} \cdot \boldsymbol{\eta}_{i j}}{\partial \boldsymbol{Q}_{i j}^{*}}\right]\left[I d+T^{-1} \operatorname{sgn}\left[\operatorname{diag}\left(\lambda_{i}\right)\right] T\right]\left(\boldsymbol{Q}_{i}^{n+1}-\boldsymbol{Q}_{i}^{n}\right) \\
& \left.+\frac{1}{2}\left[\frac{\partial \boldsymbol{F}\left(\boldsymbol{Q}_{i j}^{*}\right) \cdot \boldsymbol{\eta}_{i j}}{\partial \boldsymbol{Q}_{i j}^{*}}+\boldsymbol{B}\left(\boldsymbol{Q}_{i}^{n}\right) \frac{\partial \boldsymbol{u}_{i j}^{*} \cdot \boldsymbol{\eta}_{i j}}{\partial \boldsymbol{Q}_{i j}^{*}}\right]\left[I d-T^{-1} \operatorname{sgn}\left[\operatorname{diag}\left(\lambda_{i}\right)\right] T\right]\left(\boldsymbol{Q}_{j}^{n+1}-\boldsymbol{Q}_{j}^{n}\right)\right]=0
\end{aligned}
$$




\subsection{Extension to second order space accuracy}

Scheme (37) can be written under the form :

$$
(M \Delta \boldsymbol{Q})_{i}=-\sum_{j \in v(i)}\left\|\boldsymbol{n}_{i j}\right\| \psi\left(\boldsymbol{Q}_{i}^{n}, \boldsymbol{Q}_{j}^{n}\right)
$$

with $\Delta \boldsymbol{Q}=\boldsymbol{Q}^{n+1}-\boldsymbol{Q}^{n}$ and $M$ is the matrix defined by equation (37). This scheme is only first-order accurate in time and space. To increase the order of accuracy of the scheme, we can change in the definition of the Riemann problem (25), the interface values $\boldsymbol{Q}_{L}=\boldsymbol{Q}_{i}$ and $\boldsymbol{Q}_{R}=\boldsymbol{Q}_{j}$ by linearly reconstructed states according to a MUSCL (Monotonic Upwind Scheme for Conservation Laws) [15] procedure. However, this reconstruction will increase the bandwidth of the matrix $M$ and the linear system (38) will become more difficult to store and to solve. Therefore, although, formally the resulting scheme will be still first-order accurate, we will use instead of (38), the time-advancing scheme :

$$
(M \Delta \boldsymbol{Q})_{i}=-\sum_{j \in v(i)}\left\|\boldsymbol{n}_{i j}\right\| \psi\left(\boldsymbol{Q}_{i j}^{n}, \boldsymbol{Q}_{j i}^{n}\right)
$$

where $\boldsymbol{Q}_{i j}$ and $\boldsymbol{Q}_{j i}$ are reconstructed values on the two side of the interface between cells $i$ and $j$. Numerical experiments show that although still formally first-order accurate, this procedure result in more accurate results than the basic first-order scheme (38). The MUSCL procedure used here is the one derived for unstructured triangulation meshes in [4]. Here, instead of using the conservative variables $\boldsymbol{Q}$, we choose instead to reconstruct the primitive variables $\boldsymbol{W}={ }^{t}\left(\alpha_{1} \rho_{1}, \alpha_{2} \rho_{2}, \boldsymbol{u}, p, \alpha_{1}\right)$, thus we set :

$$
\left\{\begin{array}{c}
\boldsymbol{W}_{i j}=\boldsymbol{W}_{i}+\frac{1}{2}(\nabla \boldsymbol{W})_{i j} . \boldsymbol{i j} \\
\boldsymbol{W}_{j i}=\boldsymbol{W}_{j}-\frac{1}{2}(\nabla \boldsymbol{W})_{j i} . \boldsymbol{i j}
\end{array}\right.
$$

The approximate nodal gradients $(\nabla \boldsymbol{W})_{i j}$ and $(\nabla \boldsymbol{W})_{j i}$ are obtained using a $\beta$ combination of centered and fully upwind gradients :

$$
(\nabla \boldsymbol{W})_{i j}=(1-\beta)(\nabla \boldsymbol{W})_{i j}^{C e n t} \cdot \boldsymbol{i j}+\beta(\nabla \boldsymbol{W})_{i j}^{U p w} . \boldsymbol{i j}
$$

The centered gradient $(\nabla \boldsymbol{W})_{i j}^{\text {Cent }}$ is defined by :

$$
(\nabla \boldsymbol{W})_{i j}^{C e n t} . \boldsymbol{i j}=\boldsymbol{W}_{j}-\boldsymbol{W}_{i}
$$

The fully upwind gradient is computed according to the definition of the downstream and upstream triangles which can be associated with an edge $\left[S_{i}, S_{j}\right]$

$$
(\nabla \boldsymbol{W})_{i j}^{U p w}=\nabla \boldsymbol{W}^{G} T_{i j}
$$

RR $\mathrm{n}^{\circ} 5342$ 


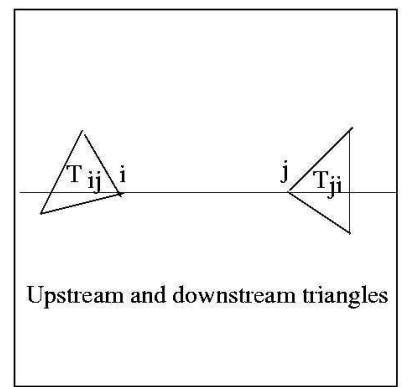

Figure 1: Definition of upwind and downwind triangle associated to edge $[\mathbf{i}, \mathbf{j}]$

where $\nabla \boldsymbol{W}^{G} T=\sum_{k \in T} \boldsymbol{W}_{k} \nabla N_{k}^{T}$ is the P1 Galerkin gradient on triangle T and where $T_{i j}$ and $T_{j i}$ are respectively the upstream and downstream triangles. (see figure 1)

$\beta$ is a parameter of upwinding included in interval $[0,1]$. In the test cases presented in the sequel, we took either $\beta=1 / 2$ or $\beta=1 / 3$. The scheme described above is not monotone. It can create extrema particularly in the case of transonic and supersonic flows. To reduce the oscillations in the solution a slope limiting procedure can be used. Here we describe two classical procedures.

Van Albada-Van Leer limiter : This limitation allows to compute an upwind coefficient $\beta^{\text {lim }} \in[0,1]$ which gives a good compromise between center and upwind gradients. The approximation writes :

$$
\left\{\begin{array}{c}
\boldsymbol{W}_{i j}=\boldsymbol{W}_{i}+\frac{1}{2} \operatorname{Lim}_{v a v l}\left((\nabla \boldsymbol{W})_{i j}^{U p w},(\nabla \boldsymbol{W})_{i j}^{C e n t}\right) \cdot \boldsymbol{i j} \\
\boldsymbol{W}_{j i}=\boldsymbol{W}_{j}-\frac{1}{2} \operatorname{Lim}_{v a v l}\left((\nabla \boldsymbol{W})_{j i}^{U p w},(\nabla \boldsymbol{W})_{j i}^{C e n t}\right) \cdot \boldsymbol{i j}
\end{array}\right.
$$

where

$$
\begin{array}{ll}
\operatorname{Lim}_{\text {vavl }}(a, b)=\beta^{l i m} a+\left(1-\beta^{l i m}\right) b & \text { if } \quad \mathrm{ab}>0 \\
\operatorname{Lim}_{\text {vavl }}(a, b)=0 & \text { if } \mathrm{ab}<0
\end{array}
$$

with

$$
\beta^{l i m}=\frac{b^{2}+\varepsilon}{a^{2}+b^{2}+2 \varepsilon}, \quad \varepsilon<<1
$$

Spekreijse limiter : This procedure uses the ratio between centered and upwind gradients. It is defined by

$$
\left\{\begin{array}{c}
\boldsymbol{W}_{i j}=\boldsymbol{W}_{i}+\frac{1}{2} \operatorname{Lim}_{\text {spek }}\left((\nabla \boldsymbol{W})_{i j}^{U p w},(\nabla \boldsymbol{W})_{i j}^{C e n t}\right) \cdot \boldsymbol{i j} \\
\boldsymbol{W}_{j i}=\boldsymbol{W}_{j}-\frac{1}{2} \operatorname{Lim}_{\text {spek }}\left((\nabla \boldsymbol{W})_{j i}^{U p w},(\nabla \boldsymbol{W})_{j i}^{C e n t}\right) \cdot \boldsymbol{i j}
\end{array}\right.
$$


where

$$
\operatorname{Lim}_{\text {spek }}(a, b)=\max \left(0, \min \left(2 \frac{b+\varepsilon}{a+\varepsilon}, \min \left(\beta+(1-\beta) \frac{b+\varepsilon}{a+\varepsilon}, 2\right)\right)\right), \quad \varepsilon<<1
$$

The Van Albada-Van Leer limiter is a little more diffusive than the Spekreijse limiter but it is robust in comparison with the Spekreijse limiter. In practice, we have used the Van Albada-Van Leer limiter with $\beta^{\text {lim }}=1 / 2$ for the implicit computations reported in this paper and the Spekreijse limiter with $\beta=1 / 3$ for the reference explicit computation done in 4.1 .

\section{Numerical results}

In this section, we present a set of two dimensional tests which show that preconditioning reduces the diffusion of the original upwind and allows to recover a better accuracy when computing low Mach number two phase flows.

\subsection{Bubble Ascension}

The first test shows the ascension of a light air-bubble under the effect of gravity in a closed box filled with water. We emphasize that a realistic simulation of this problem would have required the use of a capillarity model to take into account the effects of surface tension. However, here, our goal is not to obtain physically realistic results but instead to compare preconditioned dissipation with the classical upwind one. Initially the bubble is at rest and the pressure field has an hydrostatic profile. The box is two meters large and two meters high and the mesh is composed of $100 \times 100$ points. Although it seems simple, this computation presents several numerical difficulties. In particular, the Mach number in this computation is extremely low (it is equal to zero at time $t=0$ and increases slighly up to a value of $10^{-1}$ in the course of the computation). The density ratio between the two fluids is equal to 1000 and finally the equation of state of the two pure fluids are very different. They are :

$$
\left\{\begin{array}{llll}
p=\left(\gamma_{1}-1\right) \rho_{1} \varepsilon_{1}-\gamma_{1} \pi_{1} & \text { with } \gamma_{1}=1.4 & \text { and } \pi_{1}=0 & \text { air } \\
p=\left(\gamma_{2}-1\right) \rho_{2} \varepsilon_{2}-\gamma_{2} \pi_{2} & \text { with } \gamma_{2}=4.4 \text { and } \pi_{2}=6.10^{8} & \text { water }
\end{array}\right.
$$

This difficult test will show the effect of the preconditioning since the flow is very close to the incompressible regime. Figure 2 shows the isovalues of the volume fraction at time $=$ $0 \mathrm{~s}, 0.15 \mathrm{~s}, 0.35 \mathrm{~s}, 0.55 \mathrm{~s}, 0.75 \mathrm{~s}, 1.0 \mathrm{~s}$ for the classical upwind scheme while Figure 3 shows the results at the same times with the preconditioned numerical method. We can observe that very large differences develops during the course of the simulation. In particular, after time $t=0.55 \mathrm{~s}$, the two results have almost no ressemblance.

$\mathrm{RR} \mathrm{n}^{\circ} 5342$ 

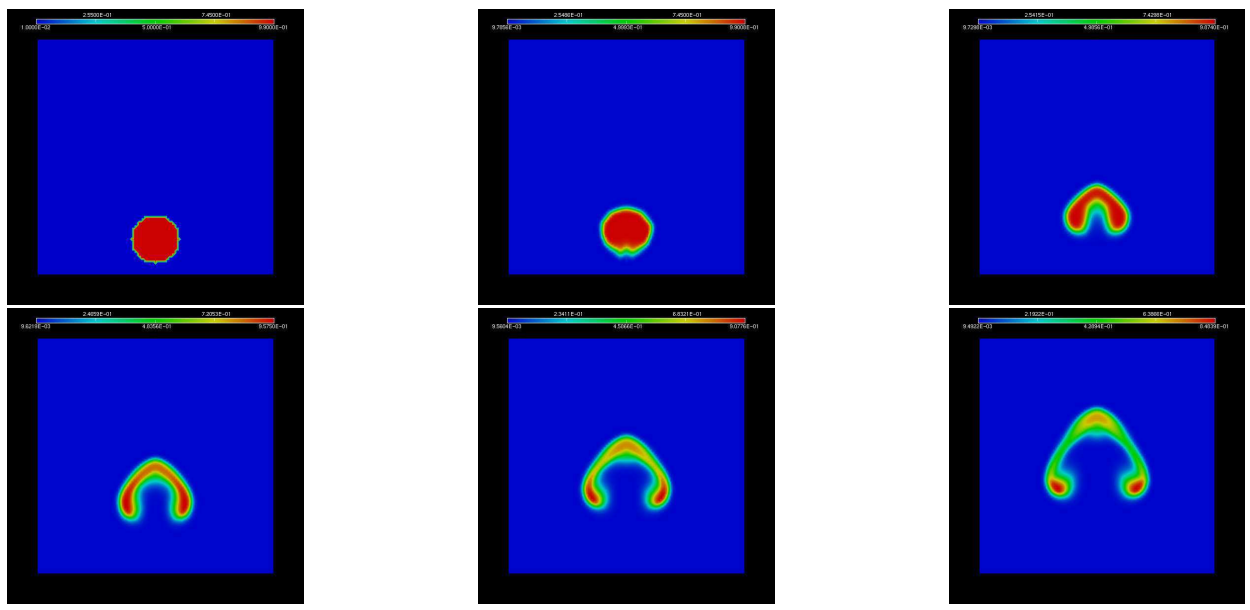

Figure 2: Bubble ascension : Isovalues of the volume fraction for the $100 \times 100$ mesh computation : classical upwind scheme
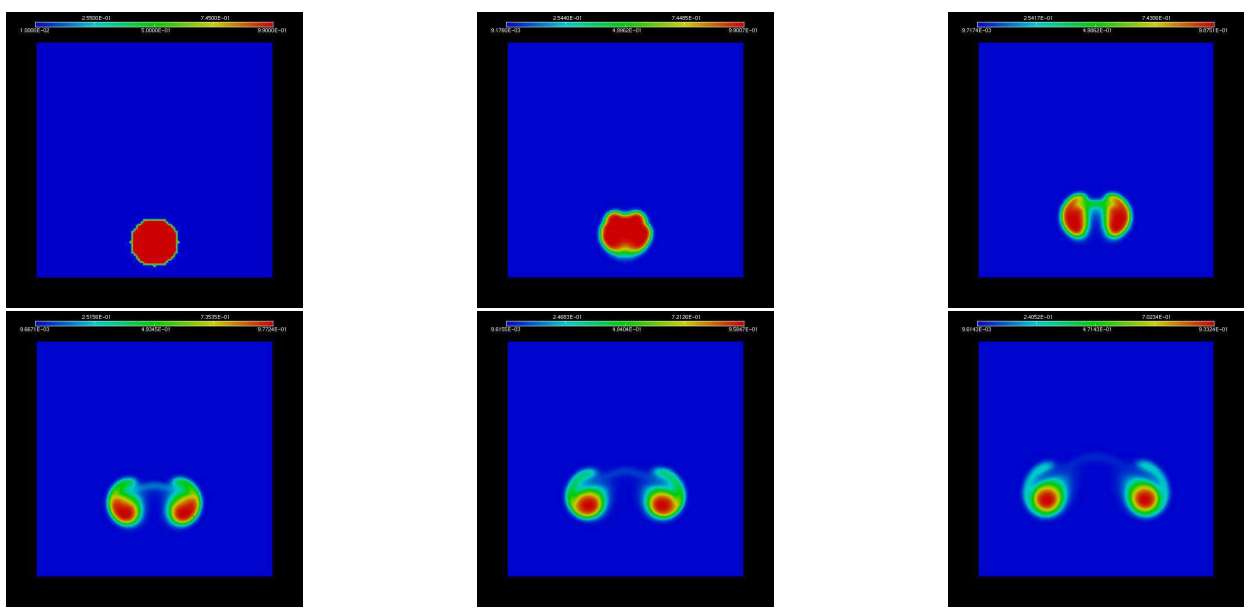

Figure 3: Bubble ascension : Isovalues of the volume fraction for the $100 \times 100$ mesh computation : preconditioned scheme 

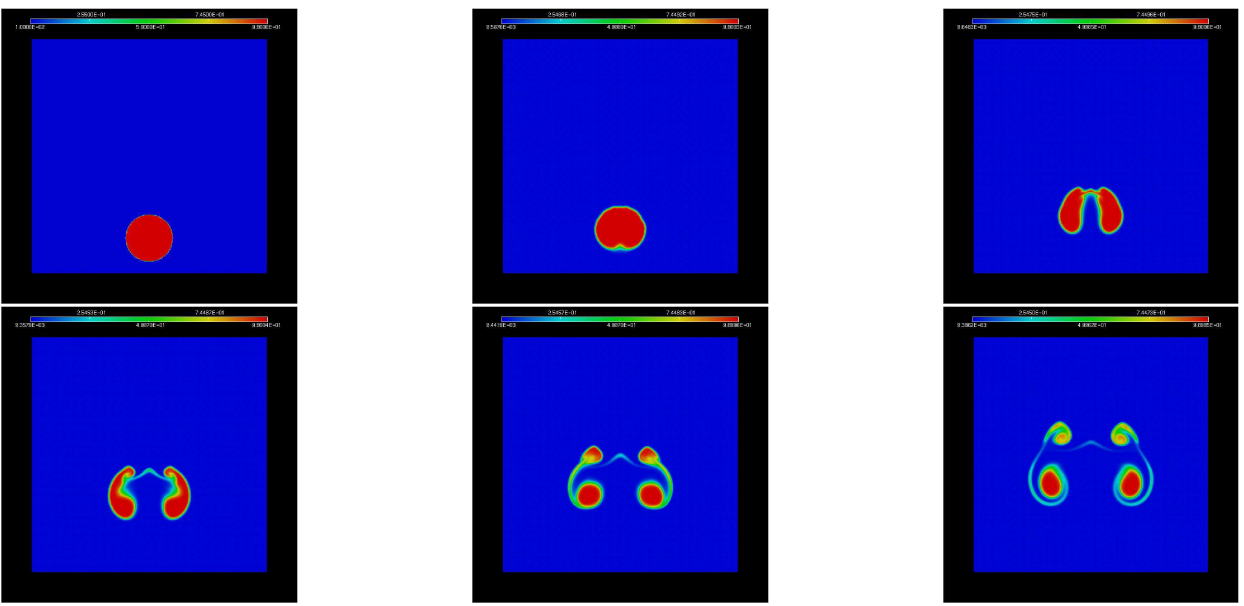

Figure 4: Bubble ascension : Isovalues of the volume fraction for the $400 \times 400$ fine mesh explicit computation

In order to demonstrate that preconditioning of the numerical dissipation improves the accuracy of the results, we have repeated this computation on a finer mesh of $400 \times 400$ points. Moreover, this fine mesh computation has been done with an explicit $2^{\text {nd }}$ order space and time scheme. Namely, the time integration has used the three stage TVD RungeKutta discretization described in [12],

$$
\left\{\begin{array}{l}
\boldsymbol{Q}_{i}^{(1)}=\boldsymbol{Q}_{i}^{n}+\Delta t \mathbf{L}\left(\boldsymbol{Q}_{i}^{n}\right) \\
\boldsymbol{Q}_{i}^{(2)}=\frac{3}{4} \boldsymbol{Q}_{i}^{n}+\frac{1}{4} \boldsymbol{Q}_{i}^{(1)}+\frac{1}{4} \Delta t \mathbf{L}\left(\boldsymbol{Q}_{i}^{(1)}\right) \\
\boldsymbol{Q}_{i}^{n+1}=\frac{1}{3} \boldsymbol{Q}_{i}^{n}+\frac{2}{3} \boldsymbol{Q}_{i}^{(2)}+\frac{2}{3} \Delta t \mathbf{L}\left(\boldsymbol{Q}_{i}^{(2)}\right)
\end{array}\right.
$$

while the space discretization has used the MUSCL technique described in section 3.3. The results of this computation are shown in Figure 4. Although, the results of this fine mesh computation are not totally identical to those of figure 3 , one can note the close similarity between these results and the one obtained with the preconditioned upwind scheme. 


\subsection{Broken Dam Problem}

Finally, this section presents a computation of the well known broken dam problem of Martin and Moyce [8]. Initially a water colummn with $a=0.06 \mathrm{~m}$ wide and $\eta^{2} a=0.12 \mathrm{~m}$ high is a rest. Under the effect of the gravity $g=9.81 \mathrm{~m} \cdot \mathrm{s}^{-2}$, the column collapses. All the boundaries are solid walls. The mesh we have used for this test, is regular with $\Delta_{x}=\Delta_{z}=5 \cdot 10^{-3} \mathrm{~m}$. The Mach number during the computation is low and of the order of $1.10^{-1}$. The implicit scheme has been used with a CFL number equal to 2.5 in order to compute with a sufficient accuracy the unsteady pattern of the flow. The linear sytem is solved by an iterative method with a linear residual $\theta=1.10^{-2}$. We compute the solution with the standard and the preconditioned method. For the preconditioned method, the parameter of the Turkel's matrix $\beta$ is chosen equal to 0.1 and remains constant in space and time.

Figures 5-6 show the isovalues of the volume fraction at the different dimensioneless time $t \sqrt{2 g / a}=0,1.19,1.98,2.97,4.02,5.09$ corresponding to the physical times $t=$ $0,0.066 \mathrm{~s}, 0.109 \mathrm{~s}, 0.164 \mathrm{~s}, 0.222 \mathrm{~s}, 0.281 \mathrm{~s}$, for the standard (figures 5) and the preconditioned method (figures 6). It is clear that the upwind preconditioned scheme predict a faster development of the flow and for instance the front position at time $0.281 \mathrm{~s}$ is clearly in advance with respect to the results obtained with the standard scheme.

To quantify the difference between the two schemes, we compare in Figure 7, the two solutions with the experimental results of [8], for the front position $x / a=F_{1}\left(\eta^{2}, t \sqrt{2 g / a}\right)$ and the height of the colummn $z /\left(\eta^{2} a\right)=F_{2}\left(\eta^{2}, t \sqrt{g / a}\right)$. It is clear that the preconditioned method is more accurate than the standard one. For example at time $t \sqrt{2 g / a}=2.97$ the error compared to the experimental data for the front position is the order of $1 \%$ for the preconditioned method while it is the order of $10 \%$ for the classical upwind scheme.

\section{Conclusion}

Since the convective part of hyperbolic models of two-phase flow derive from the one-phase Euler equations, one can suspect that a loss of accuracy will affect the results when trying to solve these models in the low Mach number regime by standard Finite Volume Upwind procedures. We have analyze this situation for a five equation two-phase flow model proposed in [5] and [7]. First, an asymptotic analysis of this model have been performed and have allowed to exhibit the equations that the solutions satisfy in the low Mach number limit. In particular, we have shown that these limit equations are very close to the incompressible single phase equations and that the pressure scale with the square of the Mach number. From a numerical point of view, we have proposed an implicit extension of the VFRoe-ncv schemes that we have apply to this model. Then using the close similarity between the mathematical structure of this two-phase model and the structure of the single phase Euler equations, we have used the strategy presented in [5] to correct the numerical dissipation by solving a preconditioned Riemann problem. The numerical results have shown that this method clearly improves the accuracy of upwind finite volume methods in the low Mach regime.

INRIA 

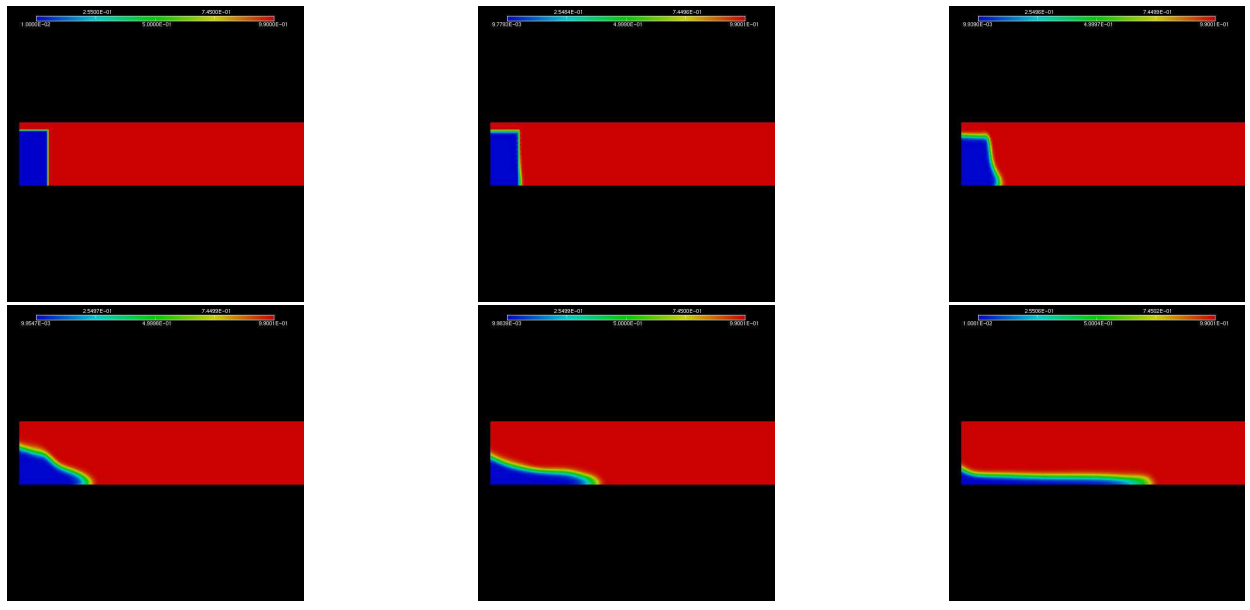

Figure 5: Broken dam problem : Isovalues of the volume fraction : classical upwind scheme
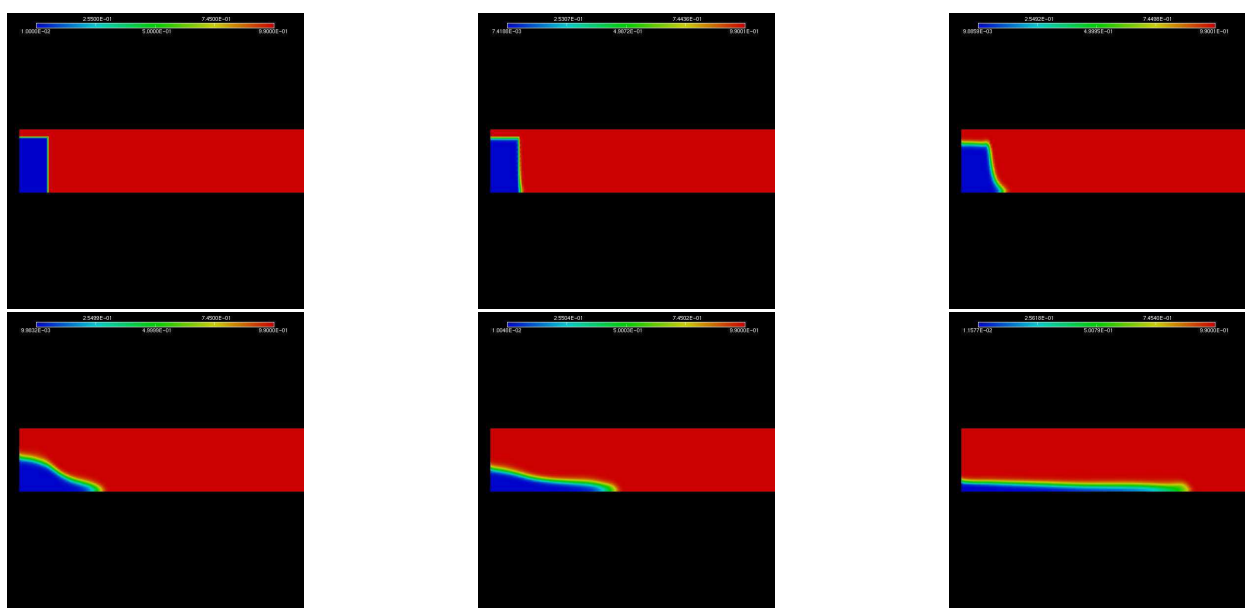

Figure 6: Broken dam problem : Isovalues of the volume fraction : preconditioned scheme 

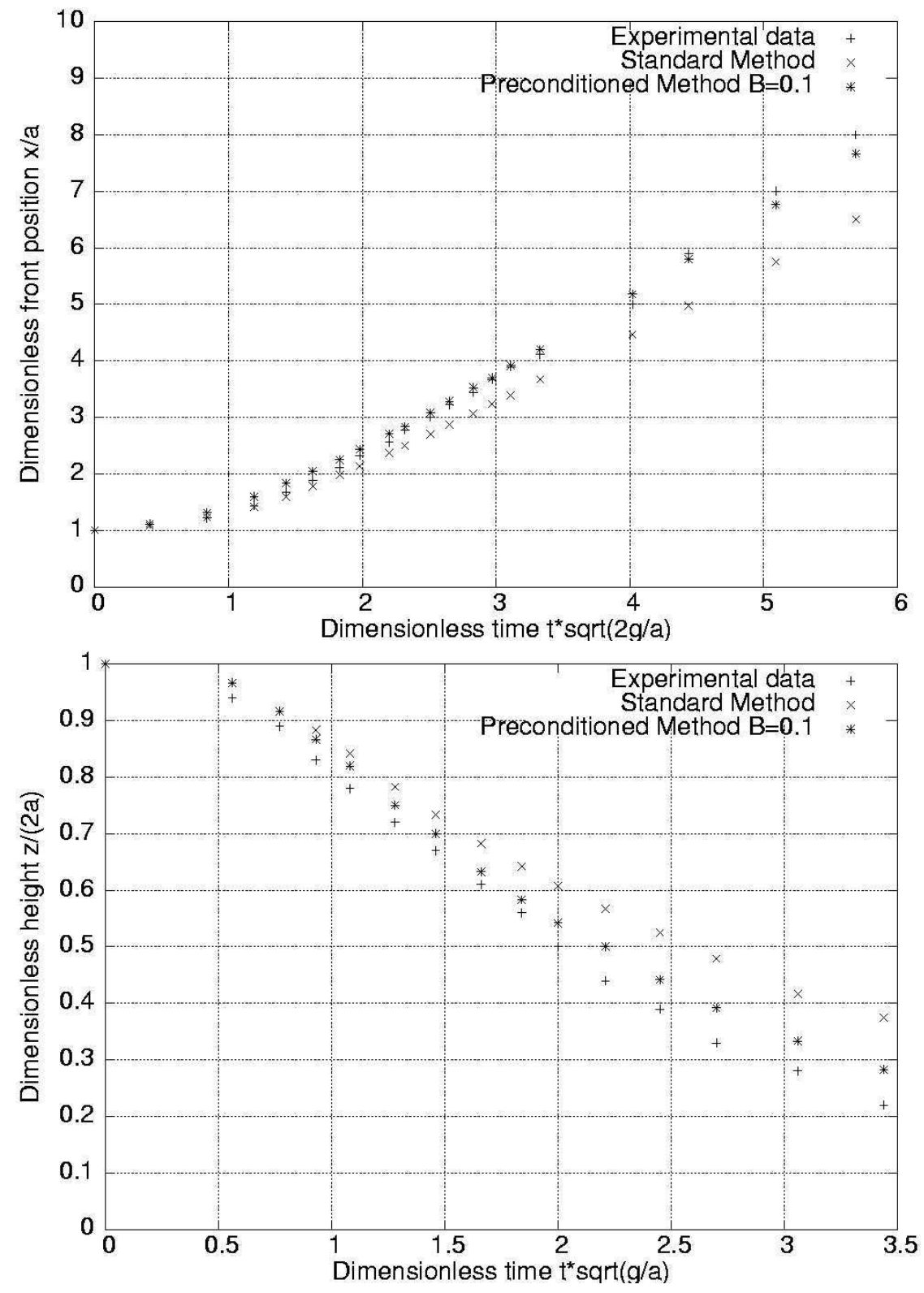

Figure 7: Comparison between numerical solutions of the classical and preconditioned scheme and experimental results for the broken dam problem. Front position (top) and height of the column (bottom). 
For instance, in the case of the broken dam problem, the error has been reduced by a factor 10 with respect to the solution obtained by a classical upwind scheme at virtually no cost.

The extension of this method to other more general two-phase flow models would be of great practical and theoretical interest since in general for two-phase flows, at least one of the fluid (the liquid) is close to the incompressible limit. However, for hyperbolic models including two velocities and two pressures, since, several Mach numbers can be defined, the situation is far from being understood even from the point of view of formal asmptotic analysis and a lot of work remains to be done.

\section{A Jacobian matrix}

The subject of this section is to compute the Jacobian matrix of model (1) and we first need to compute the differential of the pressure in term of conservative variables. So let us write $\rho_{k} \varepsilon_{k}=\rho_{k} \varepsilon_{k}\left(\rho_{k}, p\right)$ and introduce the coefficients $\delta_{k}=\left(\partial \rho_{k} \varepsilon_{k} / \partial \rho_{k}\right)_{p}$ and $\xi_{k}=\left(\partial \rho_{k} \varepsilon_{k} / \partial p\right)_{\rho_{k}}$. The differential $d\left(\rho_{k} \varepsilon_{k}\right)$ writes :

$$
d\left(\rho_{k} \varepsilon_{k}\right)=\delta_{k} d \rho_{k}+\xi_{k} d p \quad \text { for } \quad k=1,2
$$

Then we use the Gibb's relation for each phase :

$$
d \varepsilon_{k}=T_{k} d s_{k}+\frac{p_{k}}{\rho_{k}^{2}} d \rho_{k} \quad \text { for } \quad k=1,2
$$

where $T_{k}$ is the temperature of phase $k$. Introducing $h_{k}=\varepsilon_{k}+p / \rho_{k}$ which stand for the specific phase enthalpies, the Gibb's relation (52) can be re-write under the form :

$$
d\left(\rho_{k} \varepsilon_{k}\right)=\rho_{k} T_{k} d s_{k}+h_{k} d \rho_{k} \quad \text { for } \quad k=1,2
$$

Now writting equality of (53)-(51), we get the well-known relations :

$$
a_{k}^{2}=\left(\frac{\partial p}{\partial \rho_{k}}\right)_{s_{k}}=\frac{h_{k}-\delta_{k}}{\xi_{k}} \quad \text { and } \quad\left(\frac{\partial p}{\partial s_{k}}\right)_{\rho_{k}}=\frac{\rho_{k} T_{k}}{\xi_{k}} \quad \text { for } \quad k=1,2
$$

where $a_{k}$ are the phase sound speeds.

Now let us compute the differential of the pressure. Using the definition of the internal

mixture energy $\rho \varepsilon=\sum_{k=1}^{2} \alpha_{k} \rho_{k} \varepsilon_{k}$ and summing the differentials (51) for the two phases, we get after some manipulations :

$$
\sum_{k=1}^{2} \alpha_{k} \xi_{k} d p=-\sum_{k=1}^{2} \delta_{k} d\left(\alpha_{k} \rho_{k}\right)+d(\rho \varepsilon)-\sum_{k=1}^{2}\left(\rho_{k} \varepsilon_{k}-\rho_{k} \delta_{k}\right) d \alpha_{k}
$$


Now introducing the new parameter $\xi=\sum_{k=1}^{2} \alpha_{k} \xi_{k}$ and also the relation $d(\rho \varepsilon)=\boldsymbol{u}^{2} / 2 \sum_{k=1}^{2} d\left(\alpha_{k} \rho_{k}\right)-$ $\boldsymbol{u} . d(\rho \boldsymbol{u})+d(\rho e)$, we get the differential of the pressure in term of conservative variables :

$$
\left\{\begin{array}{c}
d p=\sum_{k=1}^{2} \frac{1}{\xi}\left(\frac{\boldsymbol{u}^{2}}{2}-\delta_{k}\right) d\left(\alpha_{k} \rho_{k}\right)-\frac{\boldsymbol{u}}{\xi} \cdot d(\rho \boldsymbol{u})+\frac{1}{\xi} d(\rho e) \\
+\frac{1}{\xi}\left(\rho_{2}\left(\varepsilon_{2}-\delta_{2}\right)-\rho_{1}\left(\varepsilon_{1}-\delta_{1}\right)\right) d \alpha_{1}
\end{array}\right.
$$

And the purpose is to compute, for all normalized vector $\boldsymbol{\eta}={ }^{t}\left(\eta_{x}, \eta_{y}\right)$, the eigenelements of the matrix :

$$
D_{c}(\boldsymbol{Q})=A_{c}(\boldsymbol{Q}) \eta_{x}+B_{c}(\boldsymbol{Q}) \eta_{y}
$$

So let us consider the rotation matrix $\theta$ which allows to pass from $\boldsymbol{Q}$ in the global basis to $\tilde{\boldsymbol{Q}}=\theta \boldsymbol{Q}={ }^{t}\left(\alpha_{1} \rho_{1}, \alpha_{2} \rho_{2}, \rho v_{n}, \rho v_{t}, \rho e, \alpha_{1}\right)$ in the local basis $\left(\boldsymbol{\eta}, \boldsymbol{\eta}^{\perp}\right)$.

$v_{n}, v_{t}$ are respectively normal and tangential components of the vector velocity and given by :

$$
\left\{\begin{array}{l}
v_{n}=\boldsymbol{u} . \boldsymbol{\eta} \\
v_{t}=\boldsymbol{u} . \boldsymbol{\eta}^{\perp}
\end{array}\right.
$$

After computations, the matrix $D_{c}(\boldsymbol{Q})$ can be written under the form :

$$
\left(\begin{array}{cccccc}
Y_{2} v_{n} & -Y_{1} v_{n} & Y_{1} \eta_{x} & Y_{1} \eta_{y} & 0 & 0 \\
-Y_{2} v_{n} & Y_{1} v_{n} & Y_{2} \eta_{x} & Y_{2} \eta_{y} & 0 & 0 \\
B_{1} \eta_{x}-u v_{n} & B_{2} \eta_{x}-u v_{n} & \left(1-\frac{1}{\xi}\right) u \eta_{x}+v_{n} & \left(1-\frac{1}{\xi}\right) v \eta_{x}-v_{t} & \frac{\eta_{x}}{\xi} & M \eta_{x} \\
B_{1} \eta_{y}-v v_{n} & B_{2} \eta_{y}-v v_{n} & \left(1-\frac{1}{\xi}\right) u \eta_{y}+v_{t} & \left(1-\frac{1}{\xi}\right) v \eta_{y}+v_{n} & \frac{\eta_{y}}{\xi} & M \eta_{y} \\
\left(B_{1}-H\right) v_{n} & \left(B_{2}-H\right) v_{n} & H \eta_{x}-\frac{u v_{n}}{\xi} & H \eta_{y}-\frac{v v_{n}}{\xi} & \left(1+\frac{1}{\xi}\right) v_{n} & M v_{n} \\
-A v_{n} / \rho & -A v_{n} / \rho & A \eta_{x} / \rho & A \eta_{y} / \rho & 0 & v_{n}
\end{array}\right)
$$

where $Y_{k}=\alpha_{k} \rho_{k} / \rho$ stand for the mass fractions and $H=\sum_{k=1}^{2} Y_{k} H_{k}=e+p / \rho$ with $H_{k}=h_{k}+\boldsymbol{u}^{2} / 2$ the specific total phase enthalpies. Then the other coefficients $B_{k}, M, A$ are 
defined by :

$$
\left\{\begin{aligned}
B_{k} & =\frac{1}{\xi}\left(\frac{\boldsymbol{u}^{2}}{2}-\delta_{k}\right) \quad \text { for } \quad k=1,2 \\
M & =\frac{1}{\xi}\left(\rho_{2}\left(\varepsilon_{2}-\delta_{2}\right)-\rho_{1}\left(\varepsilon_{1}-\delta_{1}\right)\right) \\
A & =\alpha_{1} \alpha_{2} \frac{\rho_{1} a_{1}^{2}-\rho_{2} a_{2}^{2}}{\sum_{k=1}^{2} \alpha_{k^{\prime}} \rho_{k} a_{k}^{2}}
\end{aligned}\right.
$$

The matrix $D_{c}(\boldsymbol{Q})$ is diagonalizable with three real distinct eigenvalues:

$$
\left\{\begin{array}{l}
\lambda_{1}(\boldsymbol{Q})=v_{n}-\hat{a} \\
\lambda_{2}(\boldsymbol{Q})=\lambda_{3}(\boldsymbol{Q})=\lambda_{4}(\boldsymbol{Q})=\lambda_{5}(\boldsymbol{Q})=v_{n} \\
\lambda_{6}(\boldsymbol{Q})=v_{n}+\hat{a}
\end{array}\right.
$$

where the value of the sound speed $\hat{a}$ is defined by the expression :

$$
\rho \hat{a}^{2}=\frac{1}{\xi} \sum_{k=1}^{2} \alpha_{k} \xi_{k} \rho_{k} a_{k}^{2}+M A
$$

which is equivalent to the averaged formula (4) given in section 2 . In effect using expressions (60.2)-(60.3) and also introducing $\rho_{2} \varepsilon_{2}-\rho_{1} \varepsilon_{1}=\rho_{2} h_{2}-\rho_{1} h_{1}$ which is only valid because the two phases have the same pressure, we get :

$$
\rho \hat{a}^{2}=\frac{1}{\xi} \sum_{k=1}^{2} \alpha_{k} \xi_{k} \rho_{k} a_{k}^{2}+\frac{1}{\xi}\left(\rho_{2}\left(h_{2}-\delta_{2}\right)-\rho_{1}\left(h_{1}-\delta_{1}\right)\right) \alpha_{1} \alpha_{2} \frac{\rho_{1} a_{1}^{2}-\rho_{2} a_{2}^{2}}{\sum_{k=1}^{2} \alpha_{k^{\prime}} \rho_{k} a_{k}^{2}}
$$

Using the relation (54) for the phase sound speed $\xi_{k} a_{k}^{2}=h_{k}-\delta_{k}$, we get :

$$
\rho \hat{a}^{2}=\frac{1}{\xi} \sum_{k=1}^{2} \alpha_{k} \xi_{k} \rho_{k} a_{k}^{2}+\frac{1}{\xi}\left(\xi_{2} \rho_{2} a_{2}^{2}-\xi_{1} \rho_{1} a_{1}^{2}\right) \alpha_{1} \alpha_{2} \frac{\rho_{1} a_{1}^{2}-\rho_{2} a_{2}^{2}}{\sum_{k=1}^{2} \alpha_{k^{\prime}} \rho_{k} a_{k}^{2}}
$$

And after manipulations, we check the following expression which is clearly equivalent to the averaged formula (4) given in section 2 :

$$
\rho \hat{a}^{2}=\frac{\rho_{1} a_{1}^{2} \rho_{2} a_{2}^{2}}{\sum_{k=1}^{2} \alpha_{k^{\prime}} \rho_{k} a_{k}^{2}}
$$

$\mathrm{RR} \mathrm{n}^{\circ} 5342$ 
Then, the right eigenvectors $r_{i}(\boldsymbol{Q})$ (for $i \in\{1, \ldots, 6\}$ ) of the matrix which verify the relation $D_{c}(\boldsymbol{Q}) r_{i}(\boldsymbol{Q})=\lambda_{i}(\boldsymbol{Q}) r_{i}(\boldsymbol{Q})$ can be choosen as :

$$
\begin{array}{rlrl}
r_{1}=\left(\begin{array}{c}
Y_{1} \\
Y_{2} \\
u-\hat{a} \eta_{x} \\
v-\hat{a} \eta_{y} \\
H-\hat{a} v_{n} \\
A / \rho
\end{array}\right) & r_{2}=\left(\begin{array}{c}
1 \\
0 \\
u \\
v \\
u^{2} / 2+\delta_{1} \\
0
\end{array}\right) r_{3}=\left(\begin{array}{c}
0 \\
1 \\
u \\
v \\
u^{2} / 2+\delta_{2} \\
0
\end{array}\right) \\
r_{4}=\left(\begin{array}{c}
0 \\
0 \\
-\eta_{y} \\
\eta_{x} \\
v_{t} \\
0
\end{array}\right) & r_{5}=\left(\begin{array}{c}
Y_{1} \\
Y_{2} \\
0 \\
0 \\
-M \xi \\
1
\end{array}\right) & r_{6}=\left(\begin{array}{c}
u \eta_{x} \\
v+\hat{a} \eta_{y} \\
H+\hat{a} v_{n} \\
A / \rho
\end{array}\right)
\end{array}
$$

We denote also by $l_{i}(\boldsymbol{Q})$ (for $i \in\{1, \ldots, 6\}$ ) the left eigenvectors which obey the relation ${ }^{t} D_{c}(\boldsymbol{Q}) l_{i}(\boldsymbol{Q})=\lambda_{i}(\boldsymbol{Q}) l_{i}(\boldsymbol{Q})$. After normalization of the left and right eigenvectors to have ${ }^{t} l_{i}(\boldsymbol{Q}) \cdot r_{j}(\boldsymbol{Q})=\delta_{i j}$, we get :

$$
\begin{gathered}
l_{1}=\frac{1}{2 \hat{a}^{2}}\left(\begin{array}{c}
B_{1}+\hat{a} v_{n} \\
B_{2}+\hat{a} v_{n} \\
-u / \xi-\hat{a} \eta_{x} \\
-v / \xi-\hat{a} \eta_{y} \\
1 / \xi \\
M
\end{array}\right) \quad l_{2}=\frac{1}{\hat{a}^{2}}\left(\begin{array}{c}
\hat{a}^{2}-Y_{1} B_{1} \\
-Y_{1} B_{2} \\
Y_{1} u / \xi \\
Y_{1} v / \xi \\
-Y_{1} / \xi \\
-Y_{1} M
\end{array}\right) \quad l_{3}=\frac{1}{\hat{a}^{2}}\left(\begin{array}{c}
-Y_{2} B_{1} \\
\hat{a}^{2}-Y_{2} B_{2} \\
Y_{2} u / \xi \\
Y_{2} v / \xi \\
-Y_{2} / \xi \\
-Y_{2} M
\end{array}\right) \\
l_{4}=\left(\begin{array}{c}
-v_{t} \\
-v_{t} \\
-\eta_{y} \\
\eta_{x} \\
0 \\
0
\end{array}\right) \quad l_{5}=\frac{1}{\rho \hat{a}^{2}}\left(\begin{array}{c}
-A B_{2} \\
B_{1}-\hat{a} v_{n} \\
B_{2}-\hat{a} v_{n} \\
-u / \xi+\hat{a} \eta_{x} \\
-v / \xi+\hat{a} \eta_{y} \\
1 / \xi \\
M \\
-A v / \xi \\
\rho \hat{a}^{2}-A M
\end{array}\right) \quad l_{6}=\frac{1}{2 \hat{a}^{2}}\left(\begin{array}{c}
\end{array}\right)
\end{gathered}
$$

\section{B Expression of the matrices $T$ and $T^{-1}$}

Here, we propose to compute the matrices $T={ }^{t}\left[l_{i}\right] R \theta$ and $T^{-1}=\theta^{-1} R^{-1}\left[r_{i}\right]$. So let us start with the computation of the matrix $R$ such as $d \boldsymbol{q}=R d \boldsymbol{Q}$. If we refer to Appendix A, 
we have :

$$
d p=\sum_{k=1}^{2} B_{k} d\left(\alpha_{k} \rho_{k}\right)-\frac{\boldsymbol{u}}{\xi} \cdot d(\rho \boldsymbol{u})+\frac{1}{\xi} d(\rho e)+M d \alpha_{1}
$$

Now it is easily checked that the differential of the velocity $\boldsymbol{u}$ is given by :

$$
d \boldsymbol{u}=-\frac{\boldsymbol{u}}{\rho} \sum_{k=1}^{2} d\left(\alpha_{k} \rho_{k}\right)+\frac{1}{\rho} d(\rho \boldsymbol{u})
$$

Then in order to compute the differential of the phase entropies $s_{k}$, we use the relation $\rho_{k} T_{k} d s_{k}=\xi_{k} d p-\xi_{k} a_{k}^{2} d \rho_{k}$ which is a consequence of (51)-(53)-(54). Introducing the differential $d p$ in this last relation, we get :

$$
\left\{\begin{aligned}
d s_{k} & =\frac{\xi_{k}}{\rho_{k} T_{k}} \sum_{k=1}^{2} B_{k} d\left(\alpha_{k} \rho_{k}\right)-\frac{\xi_{k} a_{k}^{2}}{\alpha_{k} \rho_{k} T_{k}} d\left(\alpha_{k} \rho_{k}\right)-\frac{\xi_{k} \boldsymbol{u}}{\xi \rho_{k} T_{k}} \cdot d(\rho \boldsymbol{u}) \\
& +\frac{\xi_{k}}{\xi \rho_{k} T_{k}} d(\rho e)+\frac{\xi_{k} M}{\rho_{k} T_{k}} d \alpha_{1}+\frac{\xi_{k} a_{k}^{2}}{\alpha_{k} T_{k}} d \alpha_{k} \quad \text { for } \quad k=1,2
\end{aligned}\right.
$$

Finally using the definition of mass fractions $\rho Y_{k}=\alpha_{k} \rho_{k}$, we get :

$$
\rho d Y_{1}=Y_{2} d\left(\alpha_{1} \rho_{1}\right)-Y_{1} d\left(\alpha_{2} \rho_{2}\right)
$$

which complete the computation of the matrix $R$ given by :

$$
\left.\begin{array}{cccccc}
B_{1} & B_{2} & -u / \xi & -v / \xi & 1 / \xi & M \\
-u / \rho & -u / \rho & 1 / \rho & 0 & 0 & 0 \\
-v / \rho & -v / \rho & 0 & 1 / \rho & 0 & 0 \\
\frac{\xi_{1}\left(\alpha_{1} B_{1}-a_{1}^{2}\right)}{\alpha_{1} \rho_{1} T_{1}} & \frac{\xi_{1} B_{2}}{\rho_{1} T_{1}} & -\frac{\xi_{1} u}{\xi \rho_{1} T_{1}} & -\frac{\xi_{1} v}{\xi \rho_{1} T_{1}} & \frac{\xi_{1}}{\xi \rho_{1} T_{1}} & \frac{\xi_{1} \xi_{2} a_{1}^{2} \rho_{2} a_{2}^{2}}{\xi \alpha_{1} T_{1} \rho \hat{a}^{2}} \\
\frac{\xi_{2} B_{1}}{\rho_{2} T_{2}} & \frac{\xi_{2}\left(\alpha_{2} B_{2}-a_{2}^{2}\right)}{\alpha_{2} \rho_{2} T_{2}} & -\frac{\xi_{2} u}{\xi \rho_{2} T_{2}} & -\frac{\xi_{2} v}{\xi \rho_{2} T_{2}} & \frac{\xi_{2}}{\xi \rho_{2} T_{2}} & \frac{-\xi_{1} \xi_{2} \rho_{1} a_{1}^{2} a_{2}^{2}}{\xi \alpha_{2} T_{2} \rho \hat{a}^{2}} \\
Y_{2} / \rho & -Y_{1} / \rho & 0 & 0 & 0 & 0
\end{array}\right)
$$


Now the matrix $R^{-1}$ such as $d \boldsymbol{Q}=R^{-1} d \boldsymbol{q}$ can be obtained with the same kind of manipulations and we no give more details. The matrix is given by :

$$
\left(\begin{array}{cccccc}
\frac{Y_{1}}{\hat{a}^{2}} & 0 & 0 & -\frac{\alpha_{1}^{2} \rho_{1} T_{1}}{\xi_{1} a_{1}^{2}} & -\frac{\alpha_{1} \alpha_{2} \rho_{1} T_{2}}{\xi_{2} a_{2}^{2}} & \frac{\rho^{2}}{\rho_{2}} \\
\frac{Y_{2}}{\hat{a}^{2}} & 0 & 0 & -\frac{\alpha_{1} \alpha_{2} \rho_{2} T_{1}}{\xi_{1} a_{1}^{2}} & -\frac{\alpha_{2}^{2} \rho_{2} T_{2}}{\xi_{2} a_{2}^{2}} & -\frac{\rho^{2}}{\rho_{1}} \\
\frac{u}{\hat{a}^{2}} & \rho & 0 & -\frac{\alpha_{1} \rho T_{1} u}{\xi_{1} a_{1}^{2}} & -\frac{\alpha_{2} \rho T_{2} u}{\xi_{2} a_{2}^{2}} & \rho^{2}\left(\frac{u}{\rho_{2}}-\frac{u}{\rho_{1}}\right) \\
\frac{v}{\hat{a}^{2}} & 0 & \rho & -\frac{\alpha_{1} \rho T_{1} v}{\xi_{1} a_{1}^{2}} & -\frac{\alpha_{2} \rho T_{2} v}{\xi_{2} a_{2}^{2}} & \rho^{2}\left(\frac{v}{\rho_{2}}-\frac{v}{\rho_{1}}\right) \\
\frac{H}{\hat{a}^{2}} & \rho u & \rho v & \alpha_{1} T_{1}\left(\rho_{1}-\frac{\rho H}{\xi_{1} a_{1}^{2}}\right) & \alpha_{2} T_{2}\left(\rho_{2}-\frac{\rho H}{\xi_{2} a_{2}^{2}}\right) & \rho^{2}\left(\frac{H_{1}}{\rho_{2}}-\frac{H_{2}}{\rho_{1}}\right) \\
\frac{A}{\rho \hat{a}^{2}} & 0 & 0 & \frac{\alpha_{1} \alpha_{2} T_{1}}{\xi_{1} a_{1}^{2}} & -\frac{\alpha_{1} \alpha_{2} T_{2}}{\xi_{2} a_{2}^{2}} & \frac{\rho^{2}}{\rho_{1} \rho_{2}}
\end{array}\right)
$$

Remark : The matrices $R=\partial \boldsymbol{q} / \partial \boldsymbol{Q}$ and $R^{-1}=\partial \boldsymbol{Q} / \partial \boldsymbol{q}$ are singular for $\alpha_{k}=0$ and $\alpha_{k}=1$. In practice for the numerical simulations, a pure fluid $k$ is represented by $\alpha_{k}=1-\varepsilon$ with $\varepsilon$ a small parameter. The result is not sensible to the choice of $\varepsilon$ as we have checked numerically.

To end this section, we give the expressions of matrices $T={ }^{t}\left[l_{i}\right] R \theta$ and $T^{-1}=$ $\theta^{-1} R^{-1}\left[r_{i}\right]$ which are used in the implicit numerical scheme.

The matrix $T$ writes :

$$
\left(\begin{array}{cccccc}
\frac{-r B_{1}+\beta^{2} \hat{a}^{2} v_{n}}{\sqrt{X}} & \frac{-r B_{2}+\beta^{2} \hat{a}^{2} v_{n}}{\sqrt{X}} & \frac{r u / \xi-\beta^{2} \hat{a}^{2} \eta_{x}}{\sqrt{X}} & \frac{r v / \xi-\beta^{2} \hat{a}^{2} \eta_{y}}{\sqrt{X}} & \frac{-r}{\sqrt{X} \xi} & \frac{-r M}{\sqrt{X}} \\
-v_{t} / \rho & -v_{t} / \rho & -\eta_{y} / \rho & \eta_{x} / \rho & 0 & 0 \\
\frac{\xi_{1}\left(\alpha_{1} B_{1}-a_{1}^{2}\right)}{\alpha_{1} \rho_{1} T_{1}} & \frac{\xi_{1} B_{2}}{\rho_{1} T_{1}} & \frac{-\xi_{1} u}{\xi \rho_{1} T_{1}} & \frac{-\xi_{1} v}{\xi \rho_{1} T_{1}} & \frac{\xi_{1}}{\xi \rho_{1} T_{1}} & \frac{\xi_{1} \xi_{2} a_{1}^{2} \rho_{2} a_{2}^{2}}{\alpha_{1} T_{1} \xi \rho \hat{a}^{2}} \\
\frac{\xi_{2} B_{1}}{\rho_{2} T_{2}} & \frac{\xi_{2}\left(\alpha_{2} B_{2}-a_{2}^{2}\right)}{\alpha_{2} \rho_{2} T_{2}} & \frac{-\xi_{2} u}{\xi \rho_{2} T_{2}} & \frac{-\xi_{2} v}{\xi \rho_{2} T_{2}} & \frac{\xi_{2}}{\xi \rho_{2} T_{2}} & \frac{\xi_{1} \xi_{2} \rho_{1} a_{1}^{2} a_{2}^{2}}{\alpha_{2} T_{2} \xi \rho \hat{a}^{2}} \\
Y_{2} / \rho & -Y_{1} / \rho & 0 & 0 & 0 & 0 \\
\frac{s B_{1}-\beta^{2} \hat{a}^{2} v_{n}}{\sqrt{X}} & \frac{s B_{2}-\beta^{2} \hat{a}^{2} v_{n}}{\sqrt{X}} & \frac{-s u / \xi+\beta^{2} \hat{a}^{2} \eta_{x}}{\sqrt{X}} & \frac{-s v / \xi+\beta^{2} \hat{a}^{2} \eta_{y}}{\sqrt{X}} & \frac{s}{\sqrt{X} \xi} & \frac{s M}{\sqrt{X}}
\end{array}\right)
$$


And the computation of the matrix $T^{-1}$ gives :

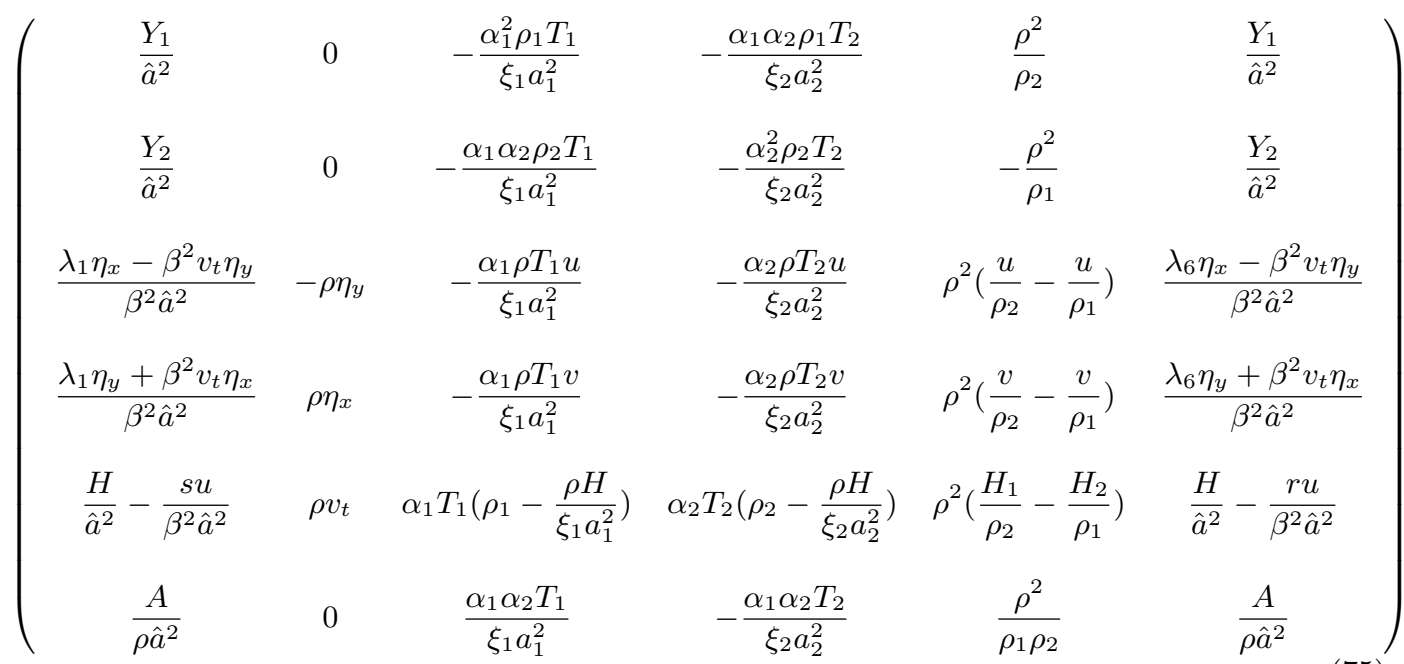

\section{References}

[1] M.R. Baer and J.W. Nunziato. A two-phase mixture theory for the deflagration-todetonation transition (DDT) in reactive granular materials. Journal of Multiphase Flows, 12:861-889, 1986.

[2] T. Buffard, T. Gallouët, and J.M. Hérard. A sequel to a Rough Godunov Scheme : Application to Real Gases. Computers and Fluids, 29:673-709, 2000.

[3] S. Clerc. Numerical Simulation of the Homogeneous Equilibrium Model for two-Phase flows. Journal of Computational Physics, 161:354-375, 2000.

[4] P-H. Cournède, C. Debiez, and A. Dervieux. A positive MUSCL scheme for triangulations. Research Report 3465, INRIA, 2002.

[5] H. Guillard and A. Murrone. On the behavior of upwind schemes in the low Mach number limit : II. Godunov type schemes. Computers and Fluids, 33:655-675, 2004.

[6] H. Guillard and C. Viozat. On the behavior of upwind schemes in the low Mach number limit. Computers and fluids, 28:63-96, 1999.

[7] A.K. Kapila, R. Menikoff, J.B. Bdzil, S.F. Son, and D.S. Stewart. Two-phase modelling of DDT in granular materials : reduced equations. Physics of Fluids, 13:3002-3024, 2001. 
[8] J.C. Martin and W.J. Moyce. An Experimental Study of the Collapse of Liquid Columns on a Rigid Horizontal Plane. Philos. Trans. Roy. Soc. London Ser A., 244:312-324, 1952.

[9] A. Murrone and H. Guillard. A five equation reduced model for compressible two phase flow problems. Journal of Computational Physics, pages -, 2004.

[10] V.H. Ransom and D.L Hicks. Hyperbolic Two-Pressure Models for Two Phase Flow. Journal of Computational Physics, 53:124-151, 1984.

[11] R. Saurel and R. Abgrall. A Multiphase Godunov Method for Compressible Multifluid and Multiphase Flows. Journal of Computational Physics, 150:425-467, 1999.

[12] C.W Shu and S. Osher. Efficient Implementation of Essential Non oscillatory Shock capturing schemes. Journal of Computational Physics, 77:439-471, 1988.

[13] H.B. Stewart and B. Wendroff. Two-phase flow : Models and methods. Journal of Computational Physics, 56:363-409, 1984.

[14] E. Turkel. Preconditioned methods for solving the incompressible and low speed compressible equations. Journal of Computational Physics, 72:277-298, 1987.

[15] B. van Leer. Towards the Ultimate Conservative Difference Scheme v. A Second-Order Sequel to Godunov's Method. Journal of Computational Physics, 32:101-136, 1979.

[16] C. Viozat. Calcul d'écoulements diphasiques dans une tuyère : Influence de la renormalisation du schéma de flux. Technical Report SYSCO/LGLS/RT/00-014, CEA, 2000.

[17] Shaojie Xu and D.S. Stewart. Deflagration-to-detonation transition in porous energetic materials: a comparative model study. Journal of Engineering Mathematics, 31:143$172,1997$. 


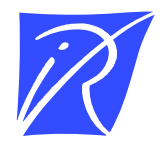

Unité de recherche INRIA Sophia Antipolis 2004, route des Lucioles - BP 93 - 06902 Sophia Antipolis Cedex (France)

Unité de recherche INRIA Futurs : Parc Club Orsay Université - ZAC des Vignes 4, rue Jacques Monod - 91893 ORSAY Cedex (France)

Unité de recherche INRIA Lorraine : LORIA, Technopôle de Nancy-Brabois - Campus scientifique 615, rue du Jardin Botanique - BP 101 - 54602 Villers-lès-Nancy Cedex (France)

Unité de recherche INRIA Rennes : IRISA, Campus universitaire de Beaulieu - 35042 Rennes Cedex (France)

Unité de recherche INRIA Rhône-Alpes : 655, avenue de l'Europe - 38334 Montbonnot Saint-Ismier (France)

Unité de recherche INRIA Rocquencourt : Domaine de Voluceau - Rocquencourt - BP 105 - 78153 Le Chesnay Cedex (France) 